\title{
Kalman Filtering Algorithm for Systems with Stochastic Nonlinearity Functions, Finite-Step Correlated Noises, and Missing Measurements
}

\author{
Yonghui He $\mathbb{D}^{1},{ }^{1}$ Jibin Jiang, ${ }^{1,2}$ Hischuan Huang, ${ }^{3}$ Shufang Zhuo, ${ }^{1}$ and Yanfeng $W u^{1}$ \\ ${ }^{1}$ Department of Mechatronics Engineering, Fujian Polytechnic of Information Technology, Fuzhou 350003, China \\ ${ }^{2}$ School of Mechanical and Automotive Engineering, Fujian University of Technology, Fuzhou 350108, China \\ ${ }^{3}$ Department of Automation Engineering, Chienkuo Technology University, Changhua 50094, Taiwan \\ Correspondence should be addressed to Yonghui He; auto_mitu@163.com
}

Received 8 August 2017; Revised 15 March 2018; Accepted 8 April 2018; Published 15 May 2018

Academic Editor: Manuel De la Sen

Copyright (C) 2018 Yonghui He et al. This is an open access article distributed under the Creative Commons Attribution License, which permits unrestricted use, distribution, and reproduction in any medium, provided the original work is properly cited.

\begin{abstract}
The locally optimal filter is designed for a class of discrete-time systems subject to stochastic nonlinearity functions, finite-step correlated noises, and missing measurements. The multiplicative noises are employed to describe the random disturbances in the system model. The phenomena of missing measurements occur in a random way and the missing probability is characterized by Bernoulli distributed random variables with known conditional probabilities. Based on the projection theory, a class of Kalman-type locally optimal filter is constructed and the filtering error covariance matrix is minimized in the sense of minimum mean square error principle. Also, by solving the recursive matrix equation, we can obtain the filter gain. Finally, two examples are provided: one is a numerical example to illustrate the feasibility and effectiveness of the proposed filtering scheme; the other is to solve the problem of target estimation for a tracking system considering networked phenomena.
\end{abstract}

\section{Introduction}

The filtering theory is a kind of optimal approach to estimate the state of the target plant based on the measurement output of the observation signals and the certain filtering criteria. Kalman filtering theory is a modern filtering theory which was proposed by Kalman in [1]. Kalman filter is an optimal estimation technique, and it is also a recursive minimum variance estimation method. From the view of geometry, the estimation of the Kalman filtering can be regarded as the projection of the state variable on the linear space generated by the observation sequence. For the linear system with the Gauss distribution noises, the recursive least mean square estimation of the system state can be obtained according to the Kalman filtering method. The state space of modern control theory is introduced into the optimal filtering theory by Kalman filtering where the dynamic model of the system is described by the equation of state and the observation equation is used to describe the observation model. In addition, the Kalman filtering can deal with the problems of time-varying systems, nonstationary signals, and multidimensional signals. Subsequently, the recursive estimation method was developed by employing the minimum mean square error principle and the projection theory. Accordingly, a considerable number of research results were reported to design the optimal filters in many practical systems [2-7].

Due to the influence of certain factors, the phenomena of correlated noises and missing measurements are inevitably encountered and the accuracy of system state estimation is degraded. Hence, the problems of correlated noises and missing measurements attracted the attention of many scholars and a large number of results were published in [8-13]. Recently, in [14], the problem of state estimation was solved for a class of linear discrete-time stochastic systems subject to missing data and correlated noises, where the estimators were unbiased and the estimation error covariances were minimized. Based on the state augmentation approach and the projection theory, in [15], the optimal linear estimator was designed in the minimum variance sense for linear uncertain systems with correlated noises and incomplete measurements 
and the sufficient condition on the existence of steady-state estimators was shown. In [16], the distributed and centralized fusion filtering problems were discussed for the sensor networked systems with stochastic uncertainties and correlated random transmission delays, where the recursive algorithms for the optimal linear distributed and centralized filters were derived by the innovation sequence analysis method. Subsequently, in [17], according to the minimax robust estimation principle, the robust centralized fusion and weighted measurement fusion time-varying Kalman estimators were presented in a unified framework for uncertain multisensor systems subject to linearly correlated white noises.

In many practical systems, the stochastic nonlinear functions are commonly encountered due to the unreliability of the communication network [18-23]. Hence, it is necessary to deal with the stochastic nonlinear functions to improve the accuracy of system state estimation. To mention but a few, in [24], a finite-horizon recursive filter was designed for discrete-time-varying nonlinear system with stochastic nonlinearities and randomly occurring measurement delays which made the filtering error minimum at each sampling time. The probability-constrained filtering problem was investigated in [25] for a class of time-varying nonlinear stochastic systems subject to estimation error variance constraint and a new filter was constructed to guarantee a minimized upper-bound on the estimation error variance. In [26], by solving two Riccati-like difference equations, an easyimplemented recursive filtering algorithm was investigated for a type of time-delayed stochastic nonlinear systems subject to event-based communication protocols and missing measurements. Based on the linear matrix inequality approach, in [25], a filter was designed to solve the problem for a class of nonlinear systems with stochastic sensor saturations and Markovian measurement transmission delays and the sufficient conditions were established to guarantee the asymptomatic stability of the filtering process. However, to the best of the authors' knowledge, these researches do not pay much attention to the problem of the optimal filtering for a class of discrete-time systems with stochastic nonlinearity functions, finite-step correlated noises, and missing measurements.

Based on the above discussions, in our paper, the purpose is to discuss the locally optimal filtering problem for a class of discrete-time systems with stochastic nonlinearity functions, finite-step correlated noises, and missing measurements. Due to the unreliable factors, the multiplicative noises are employed to describe the random disturbances in the system model. The considered phenomenon of missing measurements is modeled by a random variable satisfying Bernoulli distribution with known conditional probabilities. A class of Kalman-type locally optimal filter is designed and the filtering error covariance matrix is minimized in the sense of minimum mean square error principle by using the projection theory. By solving the recursive matrix equation, we can obtain the filter gain. Finally, two examples are given to illustrate the feasibility and effectiveness of the proposed filtering scheme. The contribution of this paper: (1) The system model is considered to add the multiplicative noises, stochastic nonlinearity, correlated noises, and missing measurements. (2) We make first attempt to design a locally optimal filter for the systems with complex network phenomena (multiplicative noises, stochastic nonlinearity functions, correlated noises, and missing measurements). (3) A new recursive algorithm is established to obtain the locally optimal filter which is suitable for online applications.

The rest of the paper is arranged as follows. In the next section, the models are proposed and a brief description of the systems is presented. In Section 3, a class of Kalman-type locally optimal filter is designed for discrete-time systems with stochastic nonlinearity functions, finite-step correlated noises, and missing measurements. In Section 4, an illustrative example is used to show the effectiveness of the proposed filtering method. Finally, the conclusions are shown in Section 5.

Notations 1 . The symbols used in the paper are standard. $\mathbb{R}^{n}$ denotes the $n$-dimensional Euclidean space. $A^{T}$ represents the transpose of matrix $A$. $\mathbb{E}\{x\}$ stands for the expectation of the variable $x . I$ and 0 represent the identity matrix and the zero matrix with appropriate dimensions, respectively. $\operatorname{diag}\left\{X_{1}, X_{2}, \ldots, X_{N}\right\}$ stands for a diagonal matrix with elements $X_{1}, X_{2}, \ldots, X_{N}$ in the diagonal. $\delta_{k-j}$ is the Kronecker delta function. If $k=j$, then $\delta_{k-j}=1$; else, $\delta_{k-j}=0$. If the dimensions of the matrices are not definitely stated, they are considered to be well-matched for algebraic operations.

\section{Problem Formulation and Preliminaries}

Consider the following discrete-time stochastic systems with multiplicative noises and stochastic nonlinearity functions:

$$
\begin{aligned}
x_{k+1} & =\left(A_{k}+A_{s, k} \epsilon_{k}\right) x_{k}+f\left(x_{k}, \eta_{k}\right)+E_{k} \omega_{k} \\
y_{k} & =\lambda_{k}\left(C_{k}+C_{s, k} \xi_{k}\right) x_{k}+g\left(x_{k}, \zeta_{k}\right)+v_{k},
\end{aligned}
$$

where $x_{k} \in \mathbb{R}^{n}$ is the state vector; $y_{k} \in \mathbb{R}^{m}$ represents the measured output. $\epsilon_{k}, \eta_{k}, \xi_{k}$, and $\zeta_{k}$ are zero-mean Gaussian white noises with unity covariances and $\epsilon_{k}, \xi_{k}$ are employed to describe the multiplicative noises, which are uncorrelated with other noise signals. The matrices $A_{k}, A_{s, k}, E_{k}, C_{k}$, and $C_{s, k}$ are known matrices with appropriate dimensions. The random variable $\lambda_{k}$, which describes the phenomenon of missing measurements, obeys the Bernoulli distribution and has the following statistical properties:

$$
\begin{aligned}
& \operatorname{Prob}\left\{\lambda_{k}=1\right\}=\mathbb{E}\left\{\lambda_{k}\right\}=\alpha, \\
& \operatorname{Prob}\left\{\lambda_{k}=0\right\}=1-\mathbb{E}\left\{\lambda_{k}\right\}=1-\alpha,
\end{aligned}
$$

where $\alpha \in[0,1]$ is a known scalar and $\lambda_{k}$ is uncorrelated with other noise signals.

In this paper, we consider the process noise $\omega_{k}$ and observation noise $v_{k}$ being correlated Gaussian white noises and satisfy the following equations:

$$
\begin{aligned}
& \mathbb{E}\left\{\omega_{k} \omega_{j}^{T}\right\}=Q_{k} \delta_{k-j}+\sum_{i=1}^{t} Q_{k, j} \delta_{k-j-i}, \\
& \mathbb{E}\left\{\nu_{k} v_{j}^{T}\right\}=R_{k} \delta_{k-j}+\sum_{i=1}^{t} R_{k, j} \delta_{k-j-i}, \\
& \mathbb{E}\left\{\omega_{k} v_{j}^{T}\right\}=S_{k} \delta_{k-j}+\sum_{i=1}^{t} S_{k, j} \delta_{k-j-i},
\end{aligned}
$$


where $Q_{k}=Q_{k}^{T}>0, R_{k}=R_{k}^{T}>0, S_{k}=S_{k}^{T}>0$, and $t$ is the number of the correlated steps. $\omega_{k}$ and $\nu_{k}$ are uncorrelated with other signals.

The functions $f\left(x_{k}, \eta_{k}\right)$ and $g\left(x_{k}, \zeta_{k}\right)$ denote the stochastic nonlinearities with $f\left(0, \epsilon_{k}\right)=0, g\left(0, \zeta_{k}\right)=0$ and satisfy the following expectations and variances for all $x_{k}$ :

$$
\begin{aligned}
\mathbb{E}\left\{f\left(x_{k}, \eta_{k}\right) \mid x_{k}\right\} & =0, \\
\mathbb{E}\left\{g\left(x_{k}, \zeta_{k}\right) \mid x_{k}\right\} & =0, \\
\mathbb{E}\left\{f\left(x_{k}, \eta_{k}\right) f\left(x_{j}, \eta_{j}\right)^{T} \mid x_{k}\right\} & =0, \\
\mathbb{E}\left\{g\left(x_{k}, \zeta_{k}\right) g\left(x_{j}, \zeta_{j}\right)^{T} \mid x_{k}\right\} & =0, \quad k \neq j, \\
\mathbb{E}\left\{f\left(x_{k}, \eta_{k}\right) f\left(x_{k}, \eta_{k}\right)^{T} \mid x_{k}\right\} & =\sum_{i=1}^{q} \Phi_{i}^{f} x_{k}^{T} \Psi^{i} x_{k}, \\
\mathbb{E}\left\{g\left(x_{k}, \zeta_{k}\right) g\left(x_{k}, \zeta_{k}\right)^{T} \mid x_{k}\right\} & =\sum_{i=1}^{q} \Phi_{i}^{g} x_{k}^{T} \Psi^{i} x_{k}, \quad k=j,
\end{aligned}
$$

where $q$ is a known nonnegative integer; $\Phi_{i}^{f}, \Phi_{i}^{g}$, and $\Psi_{i}(i=$ $1,2, \ldots, q)$ are known matrices with appropriate dimensions.

The purpose of this paper is, based on the projection theory and the minimum mean square error principle, to design the Kalman-type recursive filter as the following form:

$$
\begin{aligned}
\widehat{x}_{k+1 \mid k} & =A_{k} \widehat{x}_{k \mid k}, \\
\widehat{x}_{k+1 \mid k+1} & =\widehat{x}_{k+1 \mid k}+K_{k+1}\left(y_{k+1}-\alpha C_{k+1} \widehat{x}_{k+1 \mid k}\right),
\end{aligned}
$$

where $\widehat{x}_{k+1 \mid k+1}$ and $\widehat{x}_{k+1 \mid k}$ are the state estimation and the onestep prediction at time instant $k . K_{k+1}$ is the filter gain matrix to be determined.
Remark 2. In model (2), if $\lambda_{k}=1, y_{k}=\left(C_{k}+C_{s, k} \xi_{k}\right) x_{k}+$ $g\left(x_{k}, \zeta_{k}\right)+v_{k}$, which represents the fact that the sensor receives the data at time instant $k$ successfully and we can estimate the state by using (7) and the observation values; if $\lambda_{k}=0, y_{k}=g\left(x_{k}, \zeta_{k}\right)+v_{k}$, which stands for the fact that the sensor only receives the values of the stochastic nonlinearity function and the noises of the time instant $k$. In this case, the sensor occurs with the missing measurements and the state is estimated only by using the values of the stochastic nonlinearity function and the noises. Equation (7) of the state estimation becomes with the following form:

$$
\widehat{x}_{k+1 \mid k+1}=\widehat{x}_{k+1 \mid k}+K_{k+1}\left(g\left(x_{k+1}, \zeta_{k+1}\right)+v_{k+1}\right) .
$$

Remark 3. Due to the presence of complex network phenomena (multiplicative noises, stochastic nonlinearity functions, correlated noises, and missing measurements) in the target system model, it is difficult to explain whether the designed filter is globally optimal. The general algorithms are focused on the local solution to reduce the amount of computation and the complexity of the algorithm for complex problems. Hence, a recursive filtering algorithm is proposed to design a locally optimal filter (6)-(7) for the target plant (1)-(2) based on the observation sequence $\left\{y_{1}, y_{2}, \ldots, y_{k}\right\}$.

\section{Main Results}

In this part, we aim to derive the filter gain matrix $K_{k+1}$ to obtain the class of Kalman-type locally optimal filter by applying the projection theory and the filtering error covariance matrix $P_{k+1 \mid k+1}$ is minimized in the sense of minimum mean square error principle. Therefore, the following lemmas and definitions are introduced for promoting the subsequent derivation process.

Lemma 4. Define $L_{k, j}=\mathbb{E}\left\{x_{k-j} \omega_{k}^{T}\right\}$ and $M_{k, j}=\mathbb{E}\left\{x_{k-j} v_{k}^{T}\right\}$, where $j=0,1, \ldots, t$. Then, the following equations can be calculated:

$$
\begin{aligned}
L_{k, j}= \begin{cases}E_{k-j-1} Q_{k-j-1, k}+\sum_{i=j+2}^{t}\left(\prod_{l=j+2}^{i} A_{k+1-l}\right) E_{k-i} Q_{k-i, k}, & j=0,1, \ldots, t-2, \\
E_{k-t} Q_{k-t, k}, & j=t-1, \\
0, & j=t,\end{cases} \\
M_{k, j}= \begin{cases}E_{k-j-1} S_{k-j-1, k}+\sum_{i=j+2}^{t}\left(\prod_{l=j+2}^{i} A_{k+1-l}\right) E_{k-i} S_{k-i, k}, & j=0,1, \ldots, t-2, \\
E_{k-t} S_{k-t, k}, & j=t-1, \\
0, & j=t .\end{cases}
\end{aligned}
$$

Proof. For $j=0,1, \ldots, t-2$, based on the definition of $L_{k, j}$, it can be held as follows:

$$
\begin{aligned}
L_{k, j} & =\mathbb{E}\left\{x_{k-j} \omega_{k}^{T}\right\} \\
& =\mathbb{E}\left\{\left(\left(A_{k-j-1}+A_{s, k-j-1} \epsilon_{k-j-1}\right) x_{k-j-1}\right.\right.
\end{aligned}
$$

$$
\begin{aligned}
& \left.\left.+f\left(x_{k-j-1}, \eta_{k-j-1}\right)+E_{k-j-1} \omega_{k-j-1}\right) \omega_{k}^{T}\right\} \\
& =E_{k-j-1} \mathbb{E}\left\{\omega_{k-j-1} \omega_{k}^{T}\right\}+A_{k-j-1} \mathbb{E}\left\{x_{k-j-1} \omega_{k}^{T}\right\} \\
& +A_{s, k-j-1} \mathbb{E}\left\{\epsilon_{k-j-1} x_{k-j-1} \omega_{k}^{T}\right\}
\end{aligned}
$$




$$
\begin{aligned}
& +\mathbb{E}\left\{f\left(x_{k-j-1}, \eta_{k-j-1}\right) \omega_{k}^{T}\right\}=E_{k-j-1} Q_{k-j-1, k} \\
& +A_{k-j-1} \mathbb{E}\left\{x_{k-j-1} \omega_{k}^{T}\right\}=E_{k-j-1} Q_{k-j-1, k}+A_{k-j-1} \\
& \times \mathbb{E}\left\{\left(\left(A_{k-j-2}+A_{s, k-j-2} \epsilon_{k-j-2}\right) x_{k-j-2}\right.\right. \\
& \left.\left.+f\left(x_{k-j-2}, \eta_{k-j-2}\right)+E_{k-j-2} \omega_{k-j-2}\right) \omega_{k}^{T}\right\} \\
& =E_{k-j-1} Q_{k-j-1, k}+A_{k-j-1} E_{k-j-2} \mathbb{E}\left\{\omega_{k-j-2} \omega_{k}^{T}\right\} \\
& +A_{k-j-1} A_{k-j-2} \mathbb{E}\left\{x_{k-j-2} \omega_{k}^{T}\right\} \\
& +A_{k-j-1} A_{s, k-j-2} \mathbb{E}\left\{\epsilon_{k-j-2} x_{k-j-2} \omega_{k}^{T}\right\} \\
& +A_{k-j-1} \mathbb{E}\left\{f\left(x_{k-j-2}, \eta_{k-j-2}\right) \omega_{k}^{T}\right\} \\
& =E_{k-j-1} Q_{k-j-1, k}+A_{k-j-1} E_{k-j-2} Q_{k-j-2, k} \\
& +A_{k-j-1} A_{k-j-2} \mathbb{E}\left\{x_{k-j-2} \omega_{k}^{T}\right\}=\cdots \\
& =E_{k-j-1} Q_{k-j-1, k}+\sum_{i=j+2}^{t}\left(\prod_{l=j+2}^{i} A_{k+1-l}\right) E_{k-i} Q_{k-i, k} .
\end{aligned}
$$

Since

$$
\begin{aligned}
& \mathbb{E}\left\{x_{k-t-1} \omega_{k}^{T}\right\}=0, \\
& \mathbb{E}\left\{\omega_{k-t-1} \omega_{k}^{T}\right\}=0,
\end{aligned}
$$

then, $L_{k, t}=0$.

By using the same method in (10), we have

$$
L_{k, t-1}=E_{k-t} Q_{k-t, k}
$$

Similarly, $M_{k, j}$ can be derived.

Thus, from the above proof, (9) and (12) are true. The proof of this lemma is complete.

Lemma 5. Letting the state covariance be $X_{k+1}=$ $\mathbb{E}\left\{x_{k+1} x_{k+1}^{T}\right\}$, then one has the following equation:

$$
\begin{aligned}
X_{k+1}= & A_{k} X_{k} A_{k}^{T}+A_{k} L_{k, 0} E_{k}^{T}+A_{s, k} X_{k} A_{s, k}^{T} \\
& +\sum_{i=1}^{q} \Phi_{i}^{f} x_{k}^{T} \Psi_{i} x_{k}+E_{k} L_{k, 0}^{T} A_{k}^{T}+E_{k} Q_{k} E_{k}^{T} .
\end{aligned}
$$

Proof. By the definition of $X_{k+1}$ and Lemma 4, we can establish the following equation:

$$
\begin{gathered}
X_{k+1}=\mathbb{E}\left\{x_{k+1} x_{k+1}^{T}\right\} \\
=\mathbb{E}\left\{\left(\left(A_{k}+A_{s, k} \epsilon_{k}\right) x_{k}+f\left(x_{k}, \eta_{k}\right)+E_{k} \omega_{k}\right)\right. \\
\left.\cdot\left(\left(A_{k}+A_{s, k} \epsilon_{k}\right) x_{k}+f\left(x_{k}, \eta_{k}\right)+E_{k} \omega_{k}\right)^{T}\right\} \\
=A_{k} \mathbb{E}\left\{x_{k} x_{k}^{T}\right\} A_{k}^{T}+A_{k} \mathbb{E}\left\{x_{k} x_{k}^{T} \epsilon_{k}^{T}\right\} A_{s, k}^{T}
\end{gathered}
$$

$$
\begin{aligned}
& +A_{k} \mathbb{E}\left\{x_{k} f\left(x_{k}, \eta_{k}\right)^{T}\right\}+A_{k} \mathbb{E}\left\{x_{k} \omega_{k}^{T}\right\} E_{k}^{T} \\
& +A_{s, k} \mathbb{E}\left\{\epsilon_{k} x_{k} x_{k}^{T}\right\} A_{k}^{T}+A_{s, k} \mathbb{E}\left\{\epsilon_{k} x_{k} x_{k}^{T} \epsilon_{k}^{T}\right\} A_{s, k}^{T} \\
& +A_{s, k} \mathbb{E}\left\{\epsilon_{k} x_{k} f\left(x_{k}, \eta_{k}\right)^{T}\right\}+A_{s, k} \mathbb{E}\left\{\epsilon_{k} x_{k} \omega_{k}^{T}\right\} E_{k}^{T} \\
& +\mathbb{E}\left\{f\left(x_{k}, \eta_{k}\right) x_{k}^{T}\right\} A_{k}^{T}+\mathbb{E}\left\{f\left(x_{k}, \eta_{k}\right) x_{k}^{T} \epsilon_{k}^{T}\right\} A_{s, k}^{T} \\
& +\mathbb{E}\left\{f\left(x_{k}, \eta_{k}\right) f\left(x_{k}, \eta_{k}\right)^{T}\right\}+\mathbb{E}\left\{f\left(x_{k}, \eta_{k}\right) \omega_{k}^{T}\right\} E_{k}^{T} \\
& +E_{k} \mathbb{E}\left\{\omega_{k} x_{k}^{T}\right\} A_{k}^{T}+E_{k} \mathbb{E}\left\{\omega_{k} x_{k}^{T} \epsilon_{k}^{T}\right\} A_{s, k}^{T} \\
& +E_{k} \mathbb{E}\left\{\omega_{k} f\left(x_{k}, \eta_{k}\right)^{T}\right\}+E_{k} \mathbb{E}\left\{\omega_{k} \omega_{k}^{T}\right\} E_{k}^{T} \\
& =A_{k} X_{k} A_{k}^{T}+A_{k} L_{k, 0} E_{k}^{T}+A_{s, k} X_{k} A_{s, k}^{T} \\
& +\sum_{i=1}^{q} \Phi_{i}^{f} x_{k}^{T} \Psi_{i} x_{k}+E_{k} L_{k, 0}^{T} A_{k}^{T}+E_{k} Q_{k} E_{k}^{T} .
\end{aligned}
$$

Hence, (13) can be held. The proof of this lemma is complete.

Lemma 6. Define the matrices $\mathscr{L}_{k}=\mathbb{E}\left\{\widehat{x}_{k \mid k-1} \omega_{k}^{T}\right\}$ and $\mathscr{M}_{k}=$ $\mathbb{E}\left\{\widehat{x}_{k \mid k-1} v_{k}^{T}\right\}$. Then, the following equations are given:

$$
\begin{gathered}
\mathscr{L}_{k}=\sum_{i=1}^{t}\left(\prod_{l=1}^{i-1} A_{k-l}\left(I-\alpha K_{k-l} C_{k-l}\right)\right) \\
\cdot A_{k-i} K_{k-i}\left(\alpha C_{k-i} L_{k, i}+S_{k, k-i}^{T}\right), \\
\mathscr{M}_{k}=\sum_{i=1}^{t}\left(\prod_{l=1}^{i-1} A_{k-l}\left(I-\alpha K_{k-l} C_{k-l}\right)\right) \\
\cdot A_{k-i} K_{k-i}\left(\alpha C_{k-i} M_{k, i}+R_{k, k-i}^{\mathrm{T}}\right) .
\end{gathered}
$$

Proof. Based on (6) and (7) and Lemma 4, we have the following recursive equation:

$$
\begin{aligned}
& \mathbb{E}\left\{\widehat{x}_{k \mid k-1} \omega_{k}^{T}\right\}=\mathbb{E}\left\{A_{k-1} \widehat{x}_{k-1 \mid k-1} \omega_{k}^{T}\right\} \\
& \quad=A_{k-1} \mathbb{E}\left\{\left(\widehat{x}_{k-1 \mid k-2}\right.\right. \\
& \left.\left.+K_{k-1}\left(y_{k-1}-\alpha C_{k-1} \widehat{x}_{k-1 \mid k-2}\right)\right) \omega_{k}^{T}\right\}=A_{k-1}(I \\
& \left.\quad-\alpha K_{k-1} C_{k-1}\right) \mathbb{E}\left\{\widehat{x}_{k-1 \mid k-2} \omega_{k}^{T}\right\} \\
& +A_{k-1} K_{k-1} \mathbb{E}\left\{y_{k-1} \omega_{k}^{T}\right\}=A_{k-1}\left(I-\alpha K_{k-1} C_{k-1}\right) \\
& +\mathbb{E}\left\{A_{k-2} \widehat{x}_{k-2 \mid k-2} \omega_{k}^{T}\right\} \\
& +A_{k-1} K_{k-1} \mathbb{E}\left\{\left(\lambda_{k-1}\left(C_{k-1}+C_{s, k-1} \xi_{k-1}\right) x_{k-1}\right.\right. \\
& \left.\left.+g\left(x_{k-1}, \zeta_{k-1}\right)+v_{k-1}\right) \omega_{k}^{T}\right\}=A_{k-1}(I \\
& \left.\quad-\alpha K_{k-1} C_{k-1}\right) A_{k-2} \mathbb{E}\left\{\left(\widehat{x}_{k-2 \mid k-3}\right.\right. \\
& \left.\left.+K_{k-2}\left(y_{k-2}-\alpha C_{k-2} \widehat{x}_{k-2 \mid k-3}\right)\right) \omega_{k}^{T}\right\}
\end{aligned}
$$




$$
\begin{aligned}
& +A_{k-1} K_{k-1}\left(\alpha C_{k-1} \mathbb{E}\left\{x_{k-1} \omega_{k}^{T}\right\}\right. \\
& +\alpha C_{s, k-1} \mathbb{E}\left\{\xi_{k-1} x_{k-1} \omega_{k}^{T}\right\}+\mathbb{E}\left\{g\left(x_{k-1}, \zeta_{k-1}\right) \omega_{k}^{T}\right\} \\
& \left.+\mathbb{E}\left\{v_{k-1} \omega_{k}^{T}\right\}\right)=A_{k-1}\left(I-\alpha K_{k-1} C_{k-1}\right)\left(A_{k-2}(I\right. \\
& \left.-\alpha K_{k-2} C_{k-2}\right) \mathbb{E}\left\{\widehat{x}_{k-2 \mid k-3} \omega_{k}^{T}\right\} \\
& \left.+A_{k-2} K_{k-2} \mathbb{E}\left\{y_{k-2} \omega_{k}^{T}\right\}\right)+A_{k-1} K_{k-1}\left(\alpha C_{k-1} L_{k, 1}\right. \\
& \left.+S_{k, k-1}^{T}\right)=A_{k-1}\left(I-\alpha K_{k-1} C_{k-1}\right) A_{k-2}(I \\
& \left.-\alpha K_{k-2} C_{k-2}\right) \mathbb{E}\left\{\widehat{x}_{k-2 \mid k-3} \omega_{k}^{T}\right\}+A_{k-1}(I \\
& \left.-\alpha K_{k-1} C_{k-1}\right) A_{k-2} K_{k-2}\left(\alpha C_{k-2} L_{k, 2}+S_{k, k-2}^{T}\right) \\
& +A_{k-1} K_{k-1}\left(\alpha C_{k-1} L_{k, 1}+S_{k, k-1}^{T}\right)=\ldots \\
& =\prod_{i=1}^{t} A_{k-i}\left(I-\alpha K_{k-i} C_{k-i}\right) \mathbb{E}\left\{\widehat{x}_{k-t \mid k-t-1} \omega_{k}^{T}\right\} \\
& +\sum_{i=1}^{t}\left(\prod_{l=1}^{i-1} A_{k-l}\left(I-\alpha K_{k-l} C_{k-l}\right)\right) \\
& +A_{k-i} K_{k-i}\left(\alpha C_{k-i} L_{k, i}+S_{k, k-i}^{T}\right) .
\end{aligned}
$$

Here, we assume that $\omega_{k}$ is $t$-step correlated with the noise; then, $\mathbb{E}\left\{\widehat{x}_{k-t \mid k-t-1} \omega_{k}^{T}\right\}=0$. Thus,

$$
\begin{gathered}
\mathscr{L}_{k}=\sum_{i=1}^{t}\left(\prod_{l=1}^{i-1} A_{k-l}\left(I-\alpha K_{k-l} C_{k-l}\right)\right) \\
\cdot A_{k-i} K_{k-i}\left(\alpha C_{k-i} L_{k, i}+S_{k, k-i}^{T}\right) .
\end{gathered}
$$

Similarly, $\mathscr{M}_{k}$ can be derived. The proof of this lemma is complete.

Lemma 7. Define the one-step prediction error $\tilde{x}_{k+1 \mid k}=x_{k+1}-$ $\widehat{x}_{k+1 \mid k}$ and the filtering error $\widetilde{x}_{k+1 \mid k+1}=x_{k+1}-\widehat{x}_{k+1 \mid k+1}$. Then, the covariance matrix $P_{k+1 \mid k}=\mathbb{E}\left\{\tilde{x}_{k+1 \mid k} \tilde{x}_{k+1 \mid k}^{T}\right\}$ is computed as follows:

$$
\begin{aligned}
P_{k+1 \mid k}= & A_{k} P_{k \mid k} A_{k}^{T}+A_{k} \mathscr{H}_{k} E_{k}^{T}+A_{s, k} X_{k} A_{s, k}^{T} \\
& +\sum_{i=1}^{q} \Phi_{i}^{f} x_{k}^{T} \Psi_{i} x_{k}+E_{k} \mathscr{H}_{k}^{T} A_{k}^{T}+E_{k} Q_{k} E_{k}^{T},
\end{aligned}
$$

where

$$
\mathscr{H}_{k}=\left(I-\alpha K_{k} C_{k}\right)\left(L_{k, 0}-\mathscr{L}_{k}\right)-K_{k} S_{k}^{T} .
$$

$\mathscr{L}_{k}$ is calculated by Lemma 6 and $P_{k \mid k}=\mathbb{E}\left\{\widetilde{x}_{k \mid k} \widetilde{x}_{k \mid k}^{T}\right\}$ is the filtering error covariance.
Proof. Substituting (1) and (6) into the definition of $P_{k+1 \mid k+1}$ yields

$$
\begin{aligned}
& P_{k+1 \mid k}=\mathbb{E}\left\{\tilde{x}_{k+1 \mid k} \tilde{x}_{k+1 \mid k}^{T}\right\}=\mathbb{E}\left\{( x _ { k + 1 } - \widehat { x } _ { k + 1 | k } ) \left(x_{k+1}\right.\right. \\
& \left.\left.-\widehat{x}_{k+1 \mid k}\right)^{T}\right\} \\
& =\mathbb{E}\left\{\left(\left(\left(A_{k}+A_{s, k} \epsilon_{k}\right) x_{k}+f\left(x_{k}, \eta_{k}\right)+E_{k} \omega_{k}\right)\right.\right. \\
& \left.-A_{k} \widehat{x}_{k \mid k}\right) \\
& \times\left(\left(\left(A_{k}+A_{s, k} \epsilon_{k}\right) x_{k}+f\left(x_{k}, \eta_{k}\right)+E_{k} \omega_{k}\right)\right. \\
& \left.\left.-A_{k} \widehat{x}_{k \mid k}\right)^{T}\right\}=\mathbb{E}\left\{\left(A_{k}\left(x_{k}-\widehat{x}_{k \mid k}\right)+A_{s, k} \epsilon_{k} x_{k}\right.\right. \\
& \left.+f\left(x_{k}, \eta_{k}\right)+E_{k} \omega_{k}\right) \times\left(A_{k}\left(x_{k}-\widehat{x}_{k \mid k}\right)+A_{s, k} \epsilon_{k} x_{k}\right. \\
& \left.\left.+f\left(x_{k}, \eta_{k}\right)+E_{k} \omega_{k}\right)^{T}\right\}=\mathbb{E}\left\{\left(A_{k} \tilde{x}_{k \mid k}+A_{s, k} \epsilon_{k} x_{k}\right.\right. \\
& \left.+f\left(x_{k}, \eta_{k}\right)+E_{k} \omega_{k}\right)\left(A_{k} \tilde{x}_{k \mid k}+A_{s, k} \epsilon_{k} x_{k}\right. \\
& \left.\left.+f\left(x_{k}, \eta_{k}\right)+E_{k} \omega_{k}\right)^{T}\right\}=A_{k} \mathbb{E}\left\{\tilde{x}_{k \mid k} \tilde{x}_{k \mid k}^{T}\right\} A_{k}^{T} \\
& +A_{k} \mathbb{E}\left\{\tilde{x}_{k \mid k} x_{k}^{T} \epsilon_{k}^{T}\right\} A_{s, k}^{T}+A_{k} \mathbb{E}\left\{\tilde{x}_{k \mid k} f\left(x_{k}, \eta_{k}\right)^{T}\right\} \\
& +A_{k} \mathbb{E}\left\{\tilde{x}_{k \mid k} \omega_{k}^{T}\right\} E_{k}^{T}+A_{s, k} \mathbb{E}\left\{\epsilon_{k} x_{k} \tilde{x}_{k \mid k}^{T}\right\} A_{k}^{T} \\
& +A_{s, k} \mathbb{E}\left\{\epsilon_{k} x_{k} x_{k}^{T} \epsilon_{k}^{T}\right\} A_{s, k}^{T} \\
& +A_{s, k} \mathbb{E}\left\{\epsilon_{k} x_{k} f\left(x_{k}, \eta_{k}\right)^{T}\right\}+A_{s, k} \mathbb{E}\left\{\epsilon_{k} x_{k} \omega_{k}^{T}\right\} E_{k}^{T} \\
& +\mathbb{E}\left\{f\left(x_{k}, \eta_{k}\right) \tilde{x}_{k \mid k}^{T}\right\} A_{k}^{T}+\mathbb{E}\left\{f\left(x_{k}, \eta_{k}\right) x_{k}^{T} \epsilon_{k}^{T}\right\} A_{s, k}^{T} \\
& +\mathbb{E}\left\{f\left(x_{k}, \eta_{k}\right) f\left(x_{k}, \eta_{k}\right)^{T}\right\}+\mathbb{E}\left\{f\left(x_{k}, \eta_{k}\right) \omega_{k}^{T}\right\} E_{k}^{T} \\
& +E_{k} \mathbb{E}\left\{\omega_{k} \tilde{x}_{k \mid k}^{T}\right\} A_{k}^{T}+E_{k} \mathbb{E}\left\{\omega_{k} x_{k}^{T} \epsilon_{k}^{T}\right\} A_{s, k}^{T} \\
& +E_{k} \mathbb{E}\left\{\omega_{k} f\left(x_{k}, \eta_{k}\right)^{T}\right\}+E_{k} \mathbb{E}\left\{\omega_{k} \omega_{k}^{T}\right\} E_{k}^{T} \\
& =A_{k} P_{k \mid k} A_{k}^{T}+A_{k} \mathbb{E}\left\{\tilde{x}_{k \mid k} \omega_{k}^{T}\right\} E_{k}^{T}+A_{s, k} X_{k} A_{s, k}^{T} \\
& +\sum_{i=1}^{q} \Phi_{i}^{f} x_{k}^{T} \Psi_{i} x_{k}+E_{k} \mathbb{E}\left\{\omega_{k} \tilde{x}_{k \mid k}^{T}\right\} A_{k}^{T}+E_{k} Q_{k} E_{k}^{T} .
\end{aligned}
$$

By utilizing (7) and Lemmas 4 and 6, we have

$$
\begin{aligned}
\mathbb{E} & \left\{\tilde{x}_{k \mid k} \omega_{k}^{T}\right\}=\mathbb{E}\left\{\left(x_{k}-\widehat{x}_{k \mid k}\right) \omega_{k}^{T}\right\}=\mathbb{E}\left\{x_{k} \omega_{k}^{T}\right\} \\
& -\mathbb{E}\left\{\widehat{x}_{k \mid k} \omega_{k}^{T}\right\}=L_{k, 0} \\
& -\mathbb{E}\left\{\left(\hat{x}_{k \mid k-1}+K_{k}\left(y_{k}-\alpha C_{k} \widehat{x}_{k \mid k-1}\right)\right) \omega_{k}^{T}\right\}=L_{k, 0} \\
& -\left(I-\alpha K_{k} C_{k}\right) \mathbb{E}\left\{\widehat{x}_{k \mid k-1} \omega_{k}^{T}\right\}-K_{k} \mathbb{E}\left\{y_{k} \omega_{k}^{T}\right\}=L_{k, 0} \\
& -\left(I-\alpha K_{k} C_{k}\right) \mathscr{L}_{k}
\end{aligned}
$$




$$
\begin{aligned}
& -K_{k} \mathbb{E}\left\{\left(\lambda_{k}\left(C_{k}+C_{s, k} \xi_{k}\right) x_{k}+g\left(x_{k}, \zeta_{k}\right)+v_{k}\right) \omega_{k}^{T}\right\} \\
& =L_{k, 0}-\left(I-\alpha K_{k} C_{k}\right) \mathscr{L}_{k}-\alpha K_{k} C_{k} \mathbb{E}\left\{x_{k} \omega_{k}^{T}\right\} \\
& -\alpha K_{k} C_{s, k} \mathbb{E}\left\{\epsilon_{k} x_{k} \omega_{k}^{T}\right\}-K_{k} \mathbb{E}\left\{g\left(x_{k}, \zeta_{k}\right) \omega_{k}^{T}\right\} \\
& -K_{k} \mathbb{E}\left\{\nu_{k} \omega_{k}^{T}\right\}=L_{k, 0}-\left(I-\alpha K_{k} C_{k}\right) \mathscr{L}_{k} \\
& -\alpha K_{k} C_{k} L_{k, 0}-K_{k} S_{k}^{T}=\left(I-\alpha K_{k} C_{k}\right)\left(L_{k, 0}-\mathscr{L}_{k}\right) \\
& -K_{k} S_{k}^{T}=\mathscr{H}_{k} .
\end{aligned}
$$

Therefore, from the above proof, we can conclude that (20) and (21) are true. The proof of this lemma is complete.

Based on Lemmas 4-7, we are ready to solve the parameter problem for the Kalman-type filter (6)-(7), which makes the filtering error covariance in the sense of minimum mean square error principle at every sampling time instant.

Theorem 8. The filter gain matrix $K_{k+1}$ of the Kalman-type filter (6)-(7) is shown as follows:

$$
K_{k+1}=\left(\alpha P_{k+1 \mid k} C_{k+1}^{T}+M_{k+1,0}\right) \mathscr{E}_{k+1}^{-1}
$$

and the covariance matrix of the filtering error is computed recursively by the following equation:

$$
P_{k+1 \mid k+1}=P_{k+1 \mid k}-K_{k+1}\left(\alpha C_{k+1} P_{k+1 \mid k}+M_{k+1,0}^{T}\right),
$$

where

$$
\begin{aligned}
\mathscr{E}_{k+1}= & \alpha(1-\alpha) C_{k+1} X_{k+1} C_{k+1}^{T}+\alpha^{2} C_{k+1} P_{k+1 \mid k} C_{k+1}^{T} \\
& +\alpha C_{k+1}\left(M_{k+1,0}-\mathscr{M}_{k+1}\right) \\
& +\alpha C_{s, k+1} X_{k+1} C_{s, k+1}^{T}+\sum_{i=1}^{q} \Phi_{i}^{g} x_{k}^{T} \Psi_{i} x_{k} \\
& +\left(\alpha C_{k+1}\left(M_{k+1,0}-\mathscr{M}_{k+1}\right)\right)^{T}+R_{k+1}
\end{aligned}
$$

and the parameters $M_{k+1,0}, X_{k+1}, \mathscr{M}_{k+1}$, and $P_{k+1 \mid k}$ are calculated by Lemmas 4-7, respectively.

Proof. Based on similar method of the projection theory, we have

$$
\begin{gathered}
K_{k+1}=\mathbb{E}\left\{x_{k+1}\left(y_{k+1}-\alpha C_{k+1} \widehat{x}_{k+1 \mid k}\right)\right\} \\
\times\left(\mathbb { E } \left\{\left(y_{k+1}-\alpha C_{k+1} \widehat{x}_{k+1 \mid k}\right)\right.\right. \\
\left.\left.\cdot\left(y_{k+1}-\alpha C_{k+1} \widehat{x}_{k+1 \mid k}\right)^{T}\right\}\right)^{-1} .
\end{gathered}
$$

Substituting (2) into $y_{k+1}-\alpha C_{k+1} \widehat{x}_{k+1 \mid k}$, it has

$$
\begin{aligned}
y_{k+1}-\alpha C_{k+1} \widehat{x}_{k+1 \mid k}= & \lambda_{k+1}\left(C_{k+1}+C_{s, k+1} \xi_{k+1}\right) x_{k+1} \\
& +g\left(x_{k+1}, \zeta_{k+1}\right)+v_{k+1} \\
& -\alpha C_{k+1} \widehat{x}_{k+1 \mid k} \\
= & \left(\lambda_{k+1}-\alpha\right) C_{k+1} x_{k+1} \\
& +\alpha C_{k+1}\left(x_{k+1}-\widehat{x}_{k+1 \mid k}\right) \\
& +\lambda_{k+1} C_{s, k+1} \xi_{k+1} x_{k+1} \\
& +g\left(x_{k+1}, \zeta_{k+1}\right)+v_{k+1} \\
= & \left(\lambda_{k+1}-\alpha\right) C_{k+1} x_{k+1} \\
& +\alpha C_{k+1} \tilde{x}_{k+1 \mid k} \\
& +\lambda_{k+1} C_{s, k+1} \xi_{k+1} x_{k+1} \\
& +g\left(x_{k+1}, \zeta_{k+1}\right)+v_{k+1} .
\end{aligned}
$$

Then,

$$
\begin{aligned}
\mathbb{E} & \left\{\left(y_{k+1}-\alpha C_{k+1} \widehat{x}_{k+1 \mid k}\right)\left(y_{k+1}-\alpha C_{k+1} \widehat{x}_{k+1 \mid k}\right)^{T}\right\} \\
& =\mathbb{E}\left\{\left(\lambda_{k+1}-\alpha\right) C_{k+1} x_{k+1}+\alpha C_{k+1} \tilde{x}_{k+1 \mid k}\right. \\
& +\lambda_{k+1} C_{s, k+1} \xi_{k+1} x_{k+1}+g\left(x_{k+1}, \zeta_{k+1}\right)+v_{k+1} \\
& \times\left(\left(\lambda_{k+1}-\alpha\right) C_{k+1} x_{k+1}+\alpha C_{k+1} \tilde{x}_{k+1 \mid k}\right. \\
& \left.\left.+\lambda_{k+1} C_{s, k+1} \xi_{k+1} x_{k+1}+g\left(x_{k+1}, \zeta_{k+1}\right)+v_{k+1}\right)^{T}\right\} \\
& =C_{k+1} \mathbb{E}\left\{\left(\lambda_{k+1}-\alpha\right)^{2} x_{k+1} x_{k+1}^{T}\right\} C_{k+1}^{T}+\mathscr{D}_{1}+\mathscr{D}_{2} \\
& +\mathscr{D}_{3}+\mathscr{D}_{4}+\mathscr{D}_{1}^{T}+\alpha C_{k+1} \mathbb{E}\left\{\tilde{x}_{k+1 \mid k} \tilde{x}_{k+1 \mid k}^{T}\right\} C_{k+1}^{T} \\
& +\mathscr{D}_{5}+\mathscr{D}_{6}+\alpha C_{k+1} \mathbb{E}\left\{\tilde{x}_{k+1 \mid k} v_{k+1}^{T}\right\}+\mathscr{D}_{2}^{T}+\mathscr{D}_{5}^{T} \\
& +C_{s, k+1} \mathbb{E}\left\{\lambda_{k+1}^{2} \xi_{k+1} x_{k+1} x_{k+1}^{T} \xi_{k+1}^{T}\right\} C_{s, k+1}^{T}+\mathscr{D}_{7} \\
& +\mathscr{D}_{8}+\mathscr{D}_{3}^{T}+\mathscr{D}_{6}^{T}+\mathscr{D}_{7}^{T}+\mathbb{E}\left\{g\left(x_{k+1}, \zeta_{k+1}\right)\right. \\
& \left.\cdot g\left(x_{k+1}, \zeta_{k+1}\right)^{T}\right\}+\mathscr{D}_{9}+\mathscr{D}_{4}^{T}+\alpha \mathbb{E}\left\{v_{k+1} \tilde{x}_{k+1 \mid k}^{T}\right\} \\
& +C_{k+1}^{T}+\mathscr{D}_{8}^{T}+\mathscr{D}_{9}^{T}+\mathbb{E}\left\{v_{k+1} v_{k+1}^{T}\right\},
\end{aligned}
$$

where

$$
\begin{aligned}
& \mathscr{D}_{1}=\alpha C_{k+1} \mathbb{E}\left\{\left(\lambda_{k+1}-\alpha\right) x_{k+1} \tilde{x}_{k+1 \mid k}^{T}\right\} C_{k+1}^{T}, \\
& \mathscr{D}_{2}=C_{k+1} \mathbb{E}\left\{\lambda_{k+1}\left(\lambda_{k+1}-\alpha\right) x_{k+1} x_{k+1}^{T} \xi_{k+1}^{T}\right\} C_{s, k+1}^{T}, \\
& \mathscr{D}_{3}=C_{k+1} \mathbb{E}\left\{\left(\lambda_{k+1}-\alpha\right) x_{k+1} g\left(x_{k+1}, \zeta_{k+1}\right)^{T}\right\}, \\
& \mathscr{D}_{4}=C_{k+1} \mathbb{E}\left\{\left(\lambda_{k+1}-\alpha\right) x_{k+1} v_{k+1}^{T}\right\},
\end{aligned}
$$




$$
\begin{aligned}
& \mathscr{D}_{5}=\alpha C_{k+1} \mathbb{E}\left\{\lambda_{k+1} \tilde{x}_{k+1 \mid k} x_{k+1}^{T} \xi_{k+1}^{T}\right\} C_{s, k+1}^{T}, \\
& \mathscr{D}_{6}=\alpha C_{k+1} \mathbb{E}\left\{\tilde{x}_{k+1 \mid k} g\left(x_{k+1}, \zeta_{k+1}\right)^{T}\right\}, \\
& \mathscr{D}_{7}=C_{s, k+1} \mathbb{E}\left\{\lambda_{k+1} \xi_{k+1} x_{k+1} g\left(x_{k+1}, \zeta_{k+1}\right)^{T}\right\}, \\
& \mathscr{D}_{8}=C_{s, k+1} \mathbb{E}\left\{\lambda_{k+1} \xi_{k+1} x_{k+1} v_{k+1}^{T}\right\}, \\
& \mathscr{D}_{9}=\mathbb{E}\left\{g\left(x_{k+1}, \zeta_{k+1}\right) v_{k+1}^{T}\right\} .
\end{aligned}
$$

Since the values of the parameters $\mathscr{D}_{1}$ to $\mathscr{D}_{9}$ are equal to zero, thus,

$$
\begin{aligned}
& \mathbb{E}\left\{\left(y_{k+1}-\alpha C_{k+1} \widehat{x}_{k+1 \mid k}\right)\left(y_{k+1}-\alpha C_{k+1} \widehat{x}_{k+1 \mid k}\right)^{T}\right\} \\
& =\alpha(1-\alpha) C_{k+1} X_{k+1} C_{k+1}^{T}+\alpha^{2} C_{k+1} P_{k+1 \mid k} C_{k+1}^{T} \\
& +\alpha C_{k+1} \mathbb{E}\left\{\tilde{x}_{k+1 \mid k} v_{k+1}^{T}\right\}+\alpha C_{s, k+1} X_{k+1} C_{s, k+1} \\
& \quad+\sum_{i=1}^{q} \Phi_{i}^{g} x_{k+1}^{T} \Psi_{i} x_{k+1}+\alpha \mathbb{E}\left\{v_{k+1} \widetilde{x}_{k+1 \mid k}^{T}\right\} C_{k+1}^{T} \\
& +R_{k+1} .
\end{aligned}
$$

By using Lemmas 4 and 6, $\mathbb{E}\left\{v_{k+1} \widetilde{x}_{k+1 \mid k}^{T}\right\}$ can be computed as follows:

$$
\begin{aligned}
\mathbb{E}\left\{v_{k+1} \widetilde{x}_{k+1 \mid k}^{T}\right\} & =\mathbb{E}\left\{\left(x_{k+1}-\widehat{x}_{k+1 \mid k}\right) v_{k+1}^{T}\right\} \\
& =\mathbb{E}\left\{x_{k+1} v_{k+1}^{T}\right\}-\mathbb{E}\left\{\widehat{x}_{k+1 \mid k} v_{k+1}^{T}\right\} \\
& =M_{k+1,0}-\mathscr{M}_{k+1} .
\end{aligned}
$$

From (29) and (30), one has

$$
\begin{aligned}
\mathbb{E}\left\{\left(y_{k+1}-\alpha C_{k+1} \widehat{x}_{k+1 \mid k}\right)\left(y_{k+1}-\alpha C_{k+1} \widehat{x}_{k+1 \mid k}\right)^{T}\right\} \\
=\alpha(1-\alpha) C_{k+1} X_{k+1} C_{k+1}^{T}+\alpha^{2} C_{k+1} P_{k+1 \mid k} C_{k+1}^{T} \\
\quad+\alpha C_{k+1}\left(M_{k+1,0}-\mathscr{M}_{k+1}\right)+\alpha C_{s, k+1} X_{k+1} C_{s, k+1}^{T} \\
\quad+\sum_{i=1}^{q} \Phi_{i}^{g} x_{k}^{T} \Psi_{i} x_{k}+\left(\alpha C_{k+1}\left(M_{k+1,0}-\mathscr{M}_{k+1}\right)\right)^{T} \\
\quad+R_{k+1} \triangleq \mathscr{E}_{k+1} .
\end{aligned}
$$

On the other hand, since the fact that $\mathbb{E}\left\{\lambda_{k+1}-\alpha\right\}=0$ and $\widehat{x}_{k+1 \mid k} \perp \widetilde{x}_{k+1 \mid k}$, the following equation can be established:

$$
\begin{aligned}
& \mathbb{E}\left\{x_{k+1}\left(y_{k+1}-\alpha C_{k+1} \widehat{x}_{k+1 \mid k}\right)^{T}\right\} \\
& =\mathbb{E}\left\{x _ { k + 1 } \left(\left(\lambda_{k+1}-\alpha\right) C_{k+1} x_{k+1}+\alpha C_{k+1} \widetilde{x}_{k+1 \mid k}\right.\right. \\
& \left.\left.+\lambda_{k+1} C_{s, k+1} \xi_{k+1} x_{k+1}+g\left(x_{k+1}, \zeta_{k+1}\right)+v_{k+1}\right)^{T}\right\}
\end{aligned}
$$

$$
\begin{aligned}
& =\mathbb{E}\left\{\left(\lambda_{k+1}-\alpha\right) x_{k+1} x_{k+1}^{T}\right\} C_{k+1}^{T}+\alpha \mathbb{E}\left\{x_{k+1} \tilde{x}_{k+1 \mid k}^{T}\right\} \\
& \cdot C_{k+1}^{T}+\mathbb{E}\left\{\lambda_{k+1} x_{k+1} x_{k+1}^{T} \xi_{k+1}^{T}\right\} C_{s, k+1}^{T} \\
& +\mathbb{E}\left\{x_{k+1}^{T} g\left(x_{k+1}, \zeta_{k+1}\right)^{T}\right\}+\mathbb{E}\left\{x_{k+1} v_{k+1}^{T}\right\} \\
& =\alpha \mathbb{E}\left\{\left(\widehat{x}_{k+1 \mid k}+\tilde{x}_{k+1 \mid k}\right) \tilde{x}_{k+1 \mid k}^{T}\right\} C_{k+1}^{T}+M_{k+1,0} \\
& =\alpha \mathbb{E}\left\{\widehat{x}_{k+1 \mid k} \widetilde{x}_{k+1 \mid k}^{T}\right\} C_{k+1}^{T}+\alpha \mathbb{E}\left\{\widetilde{x}_{k+1 \mid k} \widetilde{x}_{k+1 \mid k}^{T}\right\} C_{k+1}^{T} \\
& +M_{k+1,0}=\alpha P_{k+1 \mid k} C_{k+1}^{T}+M_{k+1,0} .
\end{aligned}
$$

By (31) and (32), we can conclude that (22) and (24) are true.

Subsequently, the following derivations are shown to compute the covariance matrix of the filtering error $P_{k+1 \mid k+1}$. From the definition of $P_{k+1 \mid k+1}$ and (25), we have

$$
\begin{aligned}
P_{k+1 \mid k+1}=\mathbb{E}\left\{\tilde{x}_{k+1 \mid k+1} \tilde{x}_{k+1 \mid k+1}^{T}\right\}=\mathbb{E}\left\{\left(x_{k+1}-\widehat{x}_{k+1 \mid k+1}\right)\right. \\
\left.\cdot\left(x_{k+1}-\widehat{x}_{k+1 \mid k+1}\right)^{T}\right\} \\
=\mathbb{E}\left\{\left(x_{k+1}-\widehat{x}_{k+1 \mid k}-K_{k+1}\left(y_{k+1}-\alpha C_{k+1} \widehat{x}_{k+1 \mid k}\right)\right)\right. \\
\left.\times\left(x_{k+1}-\widehat{x}_{k+1 \mid k}-K_{k+1}\left(y_{k+1}-\alpha C_{k+1} \widehat{x}_{k+1 \mid k}\right)\right)^{T}\right\} \\
=\mathbb{E}\left\{\left(x_{k+1}-\widehat{x}_{k+1 \mid k}\right)\left(x_{k+1}-\widehat{x}_{k+1 \mid k}\right)^{T}\right\} \\
-\mathbb{E}\left\{\left(x_{k+1}-\widehat{x}_{k+1 \mid k}\right)\left(y_{k+1}-\alpha C_{k+1} \widehat{x}_{k+1 \mid k}\right)^{T}\right\} K_{k+1}^{T} \\
-K_{k+1} \mathbb{E}\left\{\left(y_{k+1}-\alpha C_{k+1} \widehat{x}_{k+1 \mid k}\right)\left(x_{k+1}-\widehat{x}_{k+1 \mid k}\right)^{T}\right\} \\
-K_{k+1} \mathbb{E}\left\{\left(y_{k+1}-\alpha C_{k+1} \widehat{x}_{k+1 \mid k}\right)\right. \\
\left.\cdot\left(y_{k+1}-\alpha C_{k+1} \widehat{x}_{k+1 \mid k}\right)^{T}\right\} K_{k+1}^{T}=\mathbb{E}\left\{\tilde{x}_{k+1 \mid k} \widetilde{x}_{k+1 \mid k}^{T}\right\} \\
-K_{k+1} \mathbb{E}\left\{\left(y_{k+1}-\alpha C_{k+1} \widehat{x}_{k+1 \mid k}\right)\left(x_{k+1}-\widehat{x}_{k+1 \mid k}\right)^{T}\right\} \\
=P_{k+1 \mid k}-K_{k+1}\left(\alpha C_{k+1} P_{k+1 \mid k}+M_{k+1,0}^{T}\right) .
\end{aligned}
$$

The proof of this theorem is complete.

Remark 9. In summary, the purpose is to obtain the optimal state estimator $\widehat{x}_{k+1 \mid k+1}$ by the following five equations:

$$
\begin{aligned}
\widehat{x}_{k+1 \mid k+1}= & \widehat{x}_{k+1 \mid k}+K_{k+1}\left(y_{k+1}-\alpha C_{k+1} \widehat{x}_{k+1 \mid k}\right), \\
K_{k+1}= & \left(\alpha P_{k+1 \mid k} C_{k+1}^{T}+M_{k+1,0}\right) \mathscr{E}_{k+1}^{-1}, \\
P_{k+1 \mid k+1}= & P_{k+1 \mid k}-K_{k+1}\left(\alpha C_{k+1} P_{k+1 \mid k}+M_{k+1,0}^{T}\right), \\
\widehat{x}_{k+1 \mid k}= & A_{k} \widehat{x}_{k \mid k}, \\
P_{k+1 \mid k}= & A_{k} P_{k \mid k} A_{k}^{T}+A_{k} \mathscr{H}_{k} E_{k}^{T}+A_{s, k} X_{k} A_{s, k}^{T} \\
& +\sum_{i=1}^{q} \Phi_{i}^{f} x_{k}^{T} \Psi_{i} x_{k}+E_{k} \mathscr{H}_{k}^{T} A_{k}^{T}+E_{k} Q_{k} E_{k}^{T},
\end{aligned}
$$


where the parameters can be computed by Lemmas 4-7. In our paper, due to considering the stochastic nonlinearity functions and finite-step correlated noises, we need to compute the values of the parameters $\sum_{i=1}^{q} \Phi_{i}^{f} x_{k}^{T} \Psi^{i} x_{k}$, $\sum_{i=1}^{q} \Phi_{i}^{g} x_{k}^{T} \Psi^{i} x_{k}$, and $L_{k, j}, M_{k, j}, \mathscr{L}_{k}$, and $\mathscr{M}_{k}$. In addition, because the phenomenon of missing measurements is taken into account, there are many cross terms in the parameter $\mathscr{E}_{k+1}$. Now, the algorithm steps of the optimal recursive filter are shown as follows.

Step 1. We can compute $L_{k, 0}$ by Lemma 4 . Then, substituting $L_{k, 0}$ and $\sum_{i=1}^{q} \Phi_{i}^{f} x_{k}^{T} \Psi^{i} x_{k}$ into (13), we can obtain $X_{k+1}$.

Step 2. Calculate $\mathscr{H}_{k}$ by substituting $L_{k, 0}$ and $\mathscr{L}_{k}$ into (21). Then, substituting $\mathscr{H}_{k}$ and $\sum_{i=1}^{q} \Phi_{i}^{f} x_{k}^{T} \Psi^{i} x_{k}$ into (20), we have $P_{k+1 \mid k}$.

Step 3. The parameter $\mathscr{E}_{k+1}$ can be computed by substituting $X_{k+1}, P_{k+1 \mid k}, M_{k+1,0}, \mathscr{M}_{k+1}$, and $\sum_{i=1}^{q} \Phi_{i}^{g} x_{k}^{T} \Psi^{i} x_{k}$ into (24).

Step 4. Substituting $P_{k+1 \mid k}, M_{k+1,0}$, and $\mathscr{E}_{k+1}$ into (22), we get $K_{k+1}$.

Step 5. The covariance matrix $P_{k+1 \mid k+1}$ of the filtering error is computed by substituting $P_{k+1 \mid k}, K_{k+1}$, and $M_{k+1,0}$ into (23).

Step 6. The optimal filter $\widehat{x}_{k+1 \mid k+1}$ is calculated by substituting (6) and (22) into (7).

Remark 10. Note that, compared with the previous results, we consider the complex network phenomena (multiplicative noises, stochastic nonlinearity functions, correlated noises, and missing measurements) and design the locally optimal filter to solve the state estimation problem for complex network systems based on the projection theory. Due to the presence of complex network phenomena in the target system model and to further improve the accuracy of filtering estimation for the systems, we compute the parameters $L_{k, j}$, $M_{k, j}, \mathscr{L}_{k}$, and $\mathscr{M}_{k}$ to deal with the finite-step correlated noises and compute the parameter $\mathscr{E}_{k+1}$ to obtain the filter gain matrix $K_{k+1}$ and the covariance matrix $P_{k+1 \mid k+1}$. Hence, the proposed filter is suitable for online applications and we make the first attempt to propose the locally optimal filter for discrete-time stochastic systems with multiplicative noises, stochastic nonlinearity functions, correlated noises, and missing measurements. Also, the filtering algorithm accuracy can be improved because we have made great efforts to compensate the effects from multiplicative noises, stochastic nonlinearity functions, correlated noises, and missing measurements.

\section{An Illustrative Example}

In this section, a numerical example is proposed to show the feasibility and effectiveness of the developed main results and a target tracking system example is given to illustrate that the proposed filter is suitable for the target tracking systems object to the complex network phenomena (multiplicative noises, stochastic nonlinearity functions, correlated noises, and missing measurements).
Example 1. Consider the following systems with multiplicative noises and stochastic nonlinearity functions:

$$
\begin{array}{r}
x_{k+1}=\left(A_{k}+A_{s, k} \epsilon_{k}\right) x_{k}+f\left(x_{k}, \eta_{k}\right)+E_{k} \omega_{k} \\
y_{k}=\lambda_{k}\left(C_{k}+C_{s, k} \xi_{k}\right) x_{k}+g\left(x_{k}, \zeta_{k}\right)+v_{k},
\end{array}
$$

where

$$
\begin{aligned}
A_{k} & =\left[\begin{array}{cc}
0.2 & -0.15 \\
0 & 0.15
\end{array}\right], \\
A_{s, k} & =\left[\begin{array}{cc}
0.01 & 0 \\
0 & 0.01
\end{array}\right], \\
E_{k} & =\left[\begin{array}{c}
2 \\
2.5
\end{array}\right], \\
C_{k} & =\left[\begin{array}{c}
1.5 \\
1
\end{array}\right]^{T}, \\
C_{s, k} & =\left[\begin{array}{c}
0.01 \\
0.01
\end{array}\right]^{T} ;
\end{aligned}
$$

$x_{k}=\left[\begin{array}{ll}x_{k}^{1} & x_{k}^{2}\end{array}\right]^{T} ; \epsilon_{k}$ and $\xi_{k}$ are uncorrelated zero-mean Gaussian white noises with unity covariances.

Here, we assumed that the noises are two-step correlated, that is, $t=2$. Then, the process noises and the observation noises can expressed as follows:

$$
\begin{aligned}
& \omega_{k}=a_{0} \varepsilon_{k}+a_{1} \varepsilon_{k-1}+a_{2} \varepsilon_{k-2}, \\
& v_{k}=b_{0} \varepsilon_{k}+b_{1} \varepsilon_{k-1}+b_{2} \varepsilon_{k-2},
\end{aligned}
$$

where $a_{0}=0.05, a_{1}=0.1$, and $a_{2}=0.15$ and $b_{0}=0.04, b_{1}=$ 0.08 , and $b_{2}=0.12 ; \varepsilon_{k}$ is uncorrelated zero-mean Gaussian white noises with unity covariances. Then, the process noises satisfy the following equation:

$$
\begin{aligned}
& \mathbb{E}\left\{\omega_{k} \omega_{k}^{T}\right\}=\mathbb{E}\left\{\left(a_{0} \varepsilon_{k}+a_{1} \varepsilon_{k-1}+a_{2} \varepsilon_{k-2}\right)\right. \\
& \left.\cdot\left(a_{0} \varepsilon_{k}+a_{1} \varepsilon_{k-1}+a_{2} \varepsilon_{k-2}\right)^{T}\right\}=a_{0}^{2} \mathbb{E}\left\{\varepsilon_{k} \varepsilon_{k}^{T}\right\} \\
& +a_{1}^{2} \mathbb{E}\left\{\varepsilon_{k-1} \varepsilon_{k-1}^{T}\right\}+a_{2}^{2} \mathbb{E}\left\{\varepsilon_{k-2} \varepsilon_{k-2}^{T}\right\}=a_{0}^{2}+a_{1}^{2}+a_{2}^{2} \\
& =Q_{k}=0.035 .
\end{aligned}
$$

Similarly, we can obtain the following results:

$$
\begin{aligned}
Q_{k, k-1} & =a_{0} a_{1}+a_{1} a_{2}=0.02, \\
Q_{k, k-2} & =a_{0} a_{2}=0.075, \\
R_{k} & =b_{0}^{2}+b_{1}^{2}+b_{2}^{2}=0.0224, \\
R_{k, k-1} & =b_{0} b_{1}+b_{1} b_{2}=0.0128,
\end{aligned}
$$




$$
\begin{aligned}
R_{k, k-2} & =b_{0} b_{2}=0.048, \\
S_{k} & =a_{0} b_{0}+a_{1} b_{1}+a_{2} b_{2}=0.028, \\
S_{k, k-1} & =a_{0} b_{1}+a_{1} b_{2}=0.016, \\
S_{k, k-2} & =a_{0} b_{2}=0.006 .
\end{aligned}
$$

We chose the stochastic nonlinear functions $f\left(x_{k}, \eta_{k}\right)$ and $g\left(x_{k}, \zeta_{k}\right)$ as follows:

$$
\begin{aligned}
& f\left(x_{k}, \eta_{k}\right) \\
& \quad=\left[\begin{array}{l}
0.1 \\
0.3
\end{array}\right]\left(0.2 \operatorname{sign}\left(x_{k}^{1}\right) x_{k}^{1} \eta_{k}^{1}+0.4 \operatorname{sign}\left(x_{k}^{2}\right) x_{k}^{2} \eta_{k}^{2}\right) \\
& g\left(x_{k}, \zeta_{k}\right) \\
& \quad=\left[\begin{array}{l}
0.2 \\
0.4
\end{array}\right]\left(0.3 \operatorname{sign}\left(x_{k}^{1}\right) x_{k}^{1} \zeta_{k}^{1}+0.5 \operatorname{sign}\left(x_{k}^{2}\right) x_{k}^{2} \zeta_{k}^{2}\right),
\end{aligned}
$$

where $x_{k}^{i}(i=1,2)$ is the $i$ th element of the system state and $\eta_{k}^{i}, \zeta_{k}^{i}$ are zero-mean Gaussian white noises with unity covariances and uncorrelated with other signals. It is easy to see that the stochastic nonlinear functions satisfy the following equations:

$$
\begin{aligned}
& \mathbb{E}\left\{f\left(x_{k}, \eta_{k}\right) \mid x_{k}\right\}=0, \\
& \mathbb{E}\left\{g\left(x_{k}, \zeta_{k}\right) \mid x_{k}\right\}=0, \\
& \mathbb{E}\left\{f\left(x_{k}, \eta_{k}\right) f\left(x_{j}, \eta_{j}\right)^{T} \mid x_{k}\right\}=0, \\
& \mathbb{E}\left\{g\left(x_{k}, \zeta_{k}\right) g\left(x_{j}, \zeta_{j}\right)^{T} \mid x_{k}\right\}=0, \quad k \neq j, \\
& \mathbb{E}\left\{f\left(x_{k}, \eta_{k}\right) f\left(x_{k}, \eta_{k}\right)^{T} \mid x_{k}\right\} \\
& \quad=\left[\begin{array}{l}
0.1 \\
0.3
\end{array}\right]\left[\begin{array}{l}
0.1 \\
0.3
\end{array}\right]^{T} x_{k}^{T} \Psi^{i} x_{k}, \\
& \mathbb{E}\left\{g\left(x_{k}, \zeta_{k}\right) g\left(x_{k}, \zeta_{k}\right)^{T} \mid x_{k}\right\} \\
& \quad=\left[\begin{array}{l}
0.2 \\
0.4
\end{array}\right]\left[\begin{array}{l}
0.2 \\
0.4
\end{array}\right]^{T} x_{k}^{T} \Psi^{i} x_{k}, \quad k=j,
\end{aligned}
$$

\begin{tabular}{|c|c|c|c|c|c|c|}
\hline$k$ & $k=0$ & $k=1$ & $k=2$ & $k=3$ & $k=4$ & \\
\hline \multirow{2}{*}{$K_{k+1}$} & 0.40 & 0.4223 & 0.3905 & 0.4262 & 0.3916 & \\
\hline & 0.4159 & 0.4612 & 0.4708 & 0.4532 & 0.4711 & \\
\hline
\end{tabular}

where $\Psi^{i}=\operatorname{diag}\{0.14,0.26\}$.

Let

$$
\begin{aligned}
x_{0} & =\left[\begin{array}{ll}
-0.1 & 0.3
\end{array}\right]^{T}, \\
\widehat{x}_{0 \mid 0} & =\left[\begin{array}{ll}
-0.2 & 0.2
\end{array}\right]^{T}, \\
P_{0 \mid 0} & =\operatorname{diag}\{1,1\}, \\
\mathbb{E}\left\{\lambda_{k}\right\} & =\alpha=0.9
\end{aligned}
$$

and MSE $i$ denote the mean square error for the estimation of $x_{k}^{i}$, that is, $(1 / M) \sum_{j=1}^{M}\left(x_{k,(j)}^{i}-\widehat{x}_{k \mid k,(j)}^{i}\right)^{2}$, where $i=1,2$ and $M$ is the number of simulation test.
TABLE 1: Filter gain matrices $K_{k+1}$.

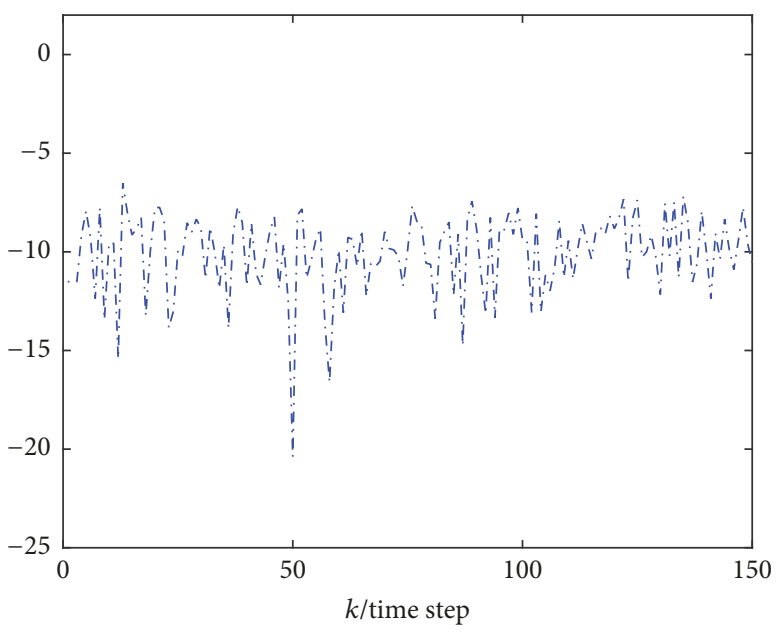

Figure 1: $\log (\mathrm{MSE} 1)$.

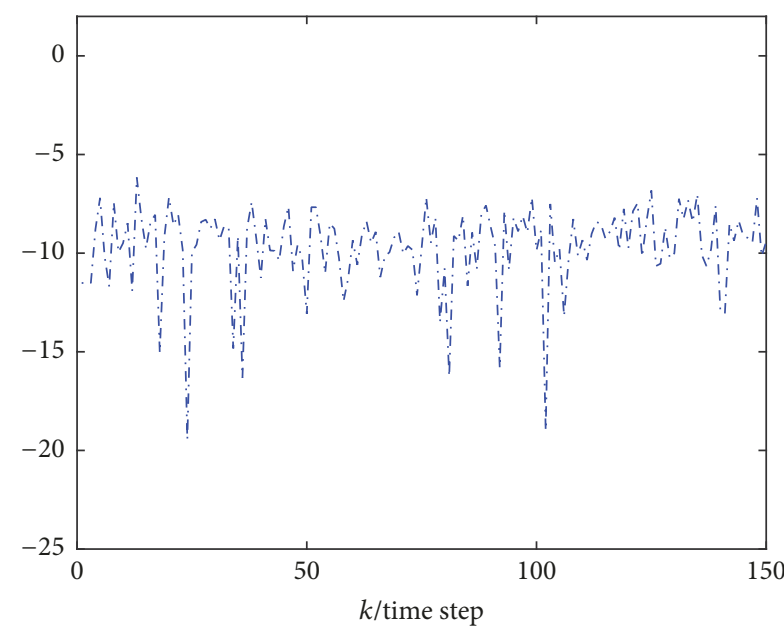

FIgURE 2: $\log ($ MSE2).

According to Theorem 8, the locally optimal filter can be constructed by applying the projection theory and the minimum mean square error estimation principle. Based on the given Kalman filtering algorithm and Matlab software, the filter gain matrices $K_{k+1}$ and the error covariance matrices $P_{k+1 \mid k+1}$ at every time step can be recursively computed and the values are shown as in Tables 1 and 2. The simulations are shown in Figures 1-6. Among them, Figures 1 and 2 plot the $\log$ (MSE- $i)(i=1,2)$ of the proposed filtering algorithm. The state of the actual system and the new designed estimation are plotted in Figures 3 and 4. Figures 5 and 6 plot the comparison between the filter in our paper and the filter without the multiplicative noises and missing measurements. From the 
TABLE 2: The error covariance matrices $P_{k+1}$.

\begin{tabular}{lccc}
\hline$k$ & $k=0$ & $k=1$ & $k=2$ \\
\hline$P_{k+1}$ & {$\left[\begin{array}{cc}0.0247 & -0.0306 \\
-0.0306 & 0.0527\end{array}\right]$} & {$\left[\begin{array}{cc}0.0023 & -0.0005 \\
-0.0005 & 0.0058\end{array}\right]$} & {$\left[\begin{array}{ll}0.0014 & 0.0016 \\
0.0016 & 0.0023\end{array}\right]$} \\
\hline$k$ & $k=3$ & $k=4$ & $\cdots$ \\
$P_{k+1}$ & {$\left[\begin{array}{ll}0.0006 & 0.0021 \\
0.0021 & 0.0019\end{array}\right]$} & {$\left[\begin{array}{ll}0.0013 & 0.0018 \\
0.0018 & 0.0020\end{array}\right]$} & $\vdots$ \\
\hline
\end{tabular}

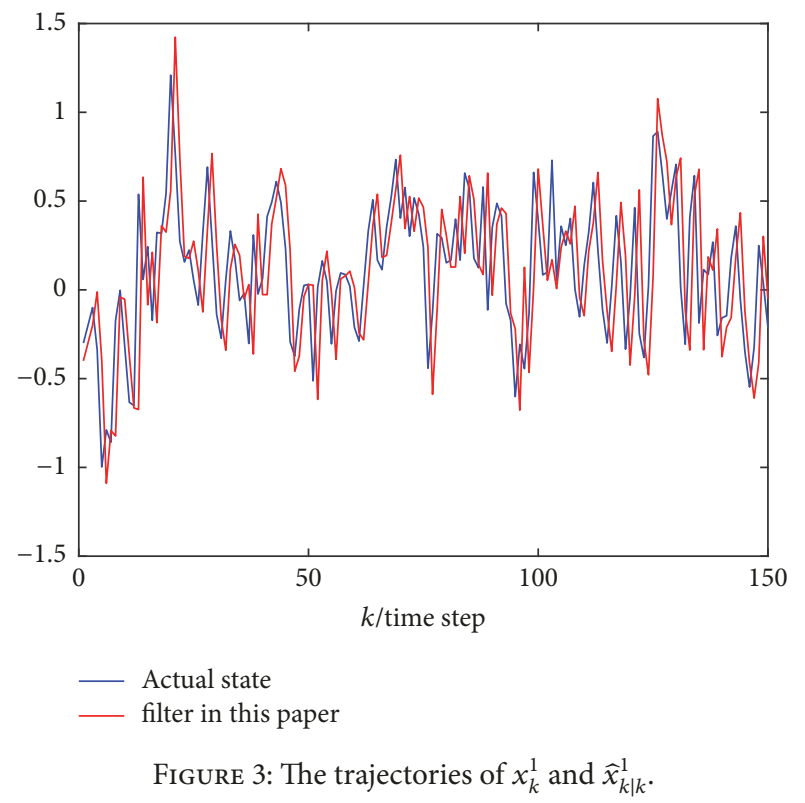

simulations, it is easily seen that the proposed filtering algorithm can estimate the system state well irrespective of the correlated noise and the occurrence of the randomly missing measurements. When deriving the locally optimal filter, the additional efforts have been made to compensate the effects from stochastic nonlinearity functions, finite-step correlated noises, and missing measurements.

Example 2. Consider the following target tracking systems with multiplicative noises and stochastic nonlinearity functions:

$$
\begin{aligned}
x_{k+1}= & \left(\left[\begin{array}{ll}
1 & T \\
0 & 1
\end{array}\right]+\left[\begin{array}{cc}
0.01 & 0 \\
0 & 0.01
\end{array}\right] \xi_{k}\right) x_{k}+f\left(x_{k}, \eta_{k}\right) \\
& +\left[\begin{array}{c}
\frac{T^{2}}{2} \\
T
\end{array}\right] \omega_{k} \\
y_{k}= & \lambda_{k}\left(\left[\begin{array}{ll}
1 & -1
\end{array}\right]+\left[\begin{array}{ll}
0.01 & 0.01
\end{array}\right] \eta_{k}\right) x_{k}+g\left(x_{k}, \zeta_{k}\right) \\
& +v_{k},
\end{aligned}
$$

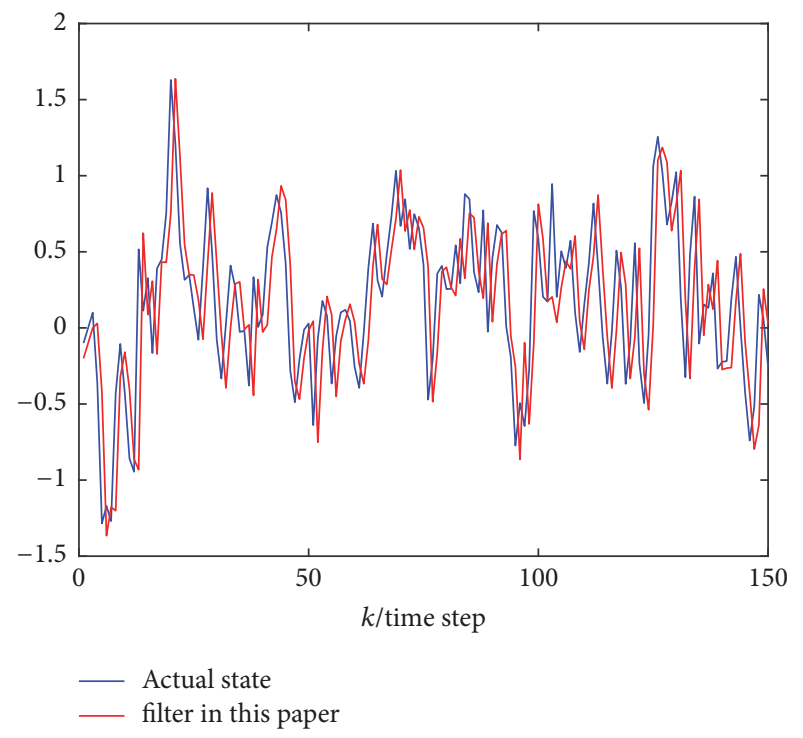

FIGURE 4: The trajectories of $x_{k}^{2}$ and $\widehat{x}_{k \mid k}^{2}$.

where $T$ is the sampling time, $T=0.5 . x_{k}=\left[\begin{array}{ll}x_{k}^{1} & x_{k}^{2}\end{array}\right]^{T}, x_{k}^{1}$ is the location, and $x_{k}^{2}$ is the velocity at time instant $k$. The other parameters are the same as those in Example 1.

The following simulation results in Figures 7 and 8 are obtained and we can see that the proposed filter, which can effectively estimate the position of the target, is suitable for the target tracking systems object to the complex network phenomena (multiplicative noises, stochastic nonlinearity functions, correlated noises, and missing measurements).

\section{Conclusion}

The problem of locally optimal filtering has been investigated for a class of discrete-time systems with stochastic nonlinearity functions, finite-step correlated noises, and missing measurements. The multiplicative noises have been employed to describe the random disturbances in the system model. Due to the unreliable factors, the phenomena of the finitestep correlated noises and missing measurements have been considered in the target plant. Based on the projection theory, a class of Kalman-type locally optimal filter is designed and the filtering error covariance matrix is minimized in the 


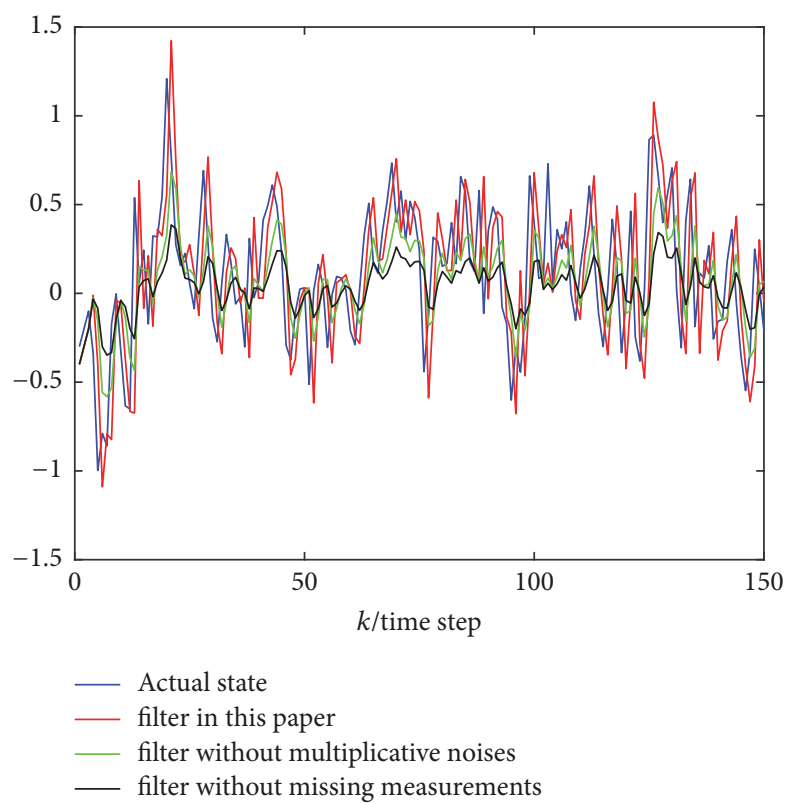

Figure 5: The trajectories of $x_{k}^{1}$.

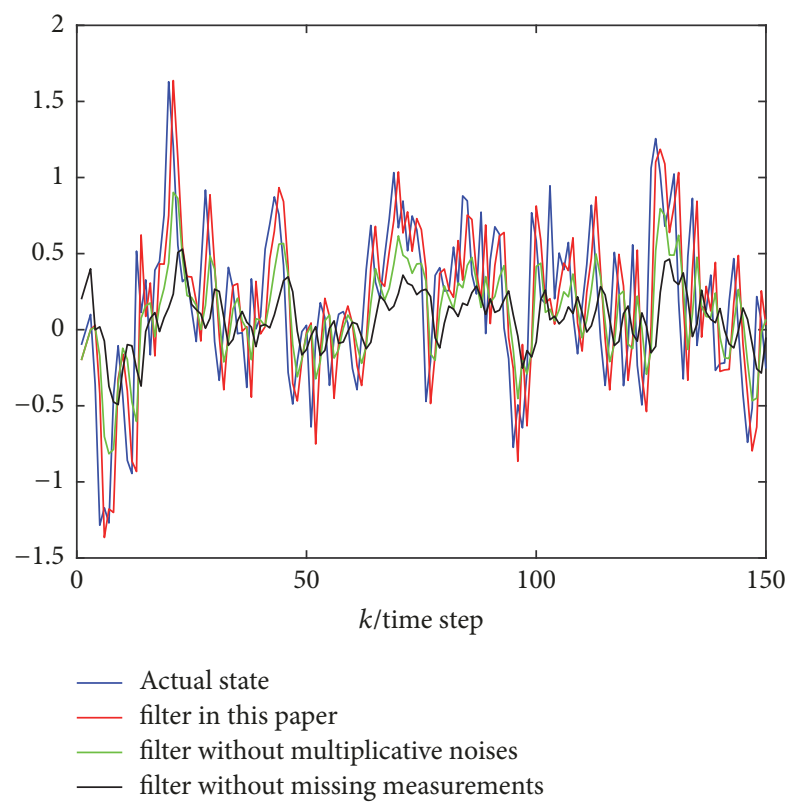

FIGURE 6: The trajectories of $x_{k}^{2}$.

sense of minimum mean square error principle. By solving the recursive matrix equation, we can obtain the filter gain. Finally, two examples are given to illustrate the feasibility and effectiveness of the proposed filtering scheme. One of the future research topics would be the stability analysis of the proposed algorithm.

\section{Conflicts of Interest}

The authors declare that there are no conflicts of interest regarding the publication of this paper.

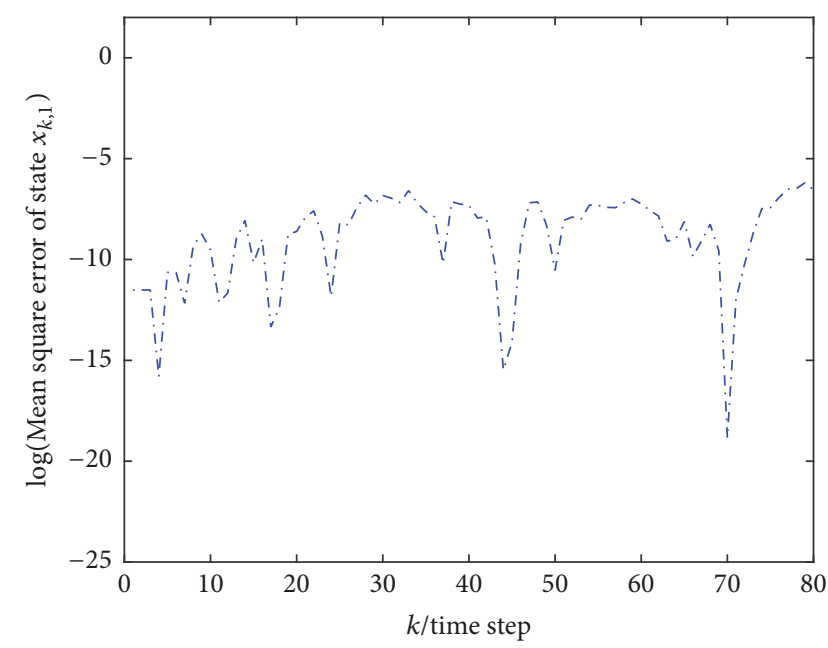

Figure 7: $\log ($ MSE1).

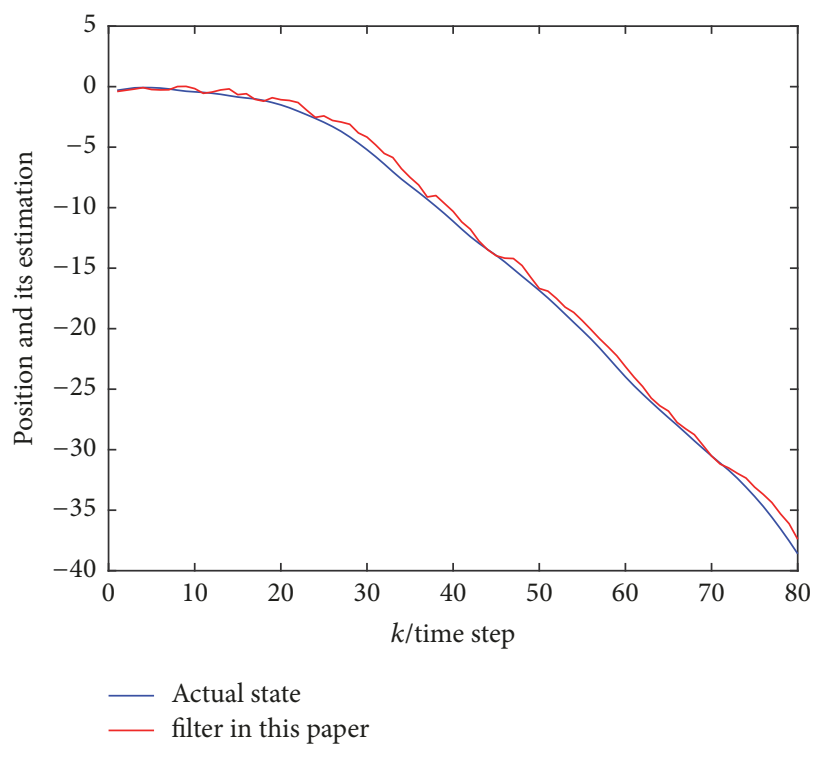

FIGURE 8: The trajectories of position.

\section{Acknowledgments}

This work was supported in part by the National Natural Science Foundation of China (NSFC) under Grant 51575110 and Fujian Young and Middle-Aged Teachers' Science and Technology Research Project under Grant JAT160725.

\section{References}

[1] R. E. Kalman, "A new approach to linear filtering and prediction problems," Journal of Fluids Engineering, vol. 82, no. 1, pp. 35-45, 1960.

[2] H. Hoely, K. J. H. Lawz, and R. Temponex, "Multilevel ensemble Kalman filtering," SIAM Journal on Numerical Analysis, vol. 54, no. 3, pp. 1813-1839, 2016.

[3] Z. Cao, R. Zhang, Y. Yang, J. Lu, and F. Gao, "DiscreteTime Robust Iterative Learning Kalman Filtering for Repetitive 
Processes," IEEE Transactions on Automatic Control, vol. 61, no. 1, pp. 270-275, 2016.

[4] V. Stojanovic and N. Nedic, "Robust Kalman filtering for nonlinear multivariable stochastic systems in the presence of nonGaussian noise," International Journal of Robust and Nonlinear Control, vol. 26, no. 3, pp. 445-460, 2016.

[5] T. Soubdhan, J. Ndong, H. Ould-Baba, and M.-T. Do, "A robust forecasting framework based on the Kalman filtering approach with a twofold parameter tuning procedure: Application to solar and photovoltaic prediction," Solar Energy, vol. 131, pp. 246-259, 2016.

[6] S. Liu, G. Wei, Y. Song, and Y. Liu, "Extended Kalman filtering for stochastic nonlinear systems with randomly occurring cyber attacks," Neurocomputing, 2016.

[7] X. Gao, L. Mo, Z. Xiao, X. Chen, and S. Katayama, "Seam tracking based on kalman filtering of micro-gap weld using magneto-optical image," The International Journal of Advanced Manufacturing Technology, vol. 83, no. 1-4, pp. 21-32, 2016.

[8] W. Li, Y. Jia, and J. Du, "Tobit Kalman filter with time-correlated multiplicative measurement noise," IET Control Theory \& Applications, vol. 11, no. 1, pp. 122-128, 2017.

[9] T. Tian, S. Sun, and N. Li, "Multi-sensor information fusion estimators for stochastic uncertain systems with correlated noises," Information Fusion, vol. 27, pp. 126-137, 2016.

[10] D. Chen, L. Xu, and J. Du, "Optimal filtering for systems with finite-step autocorrelated process noises, random one-step sensor delay and missing measurements," Communications in Nonlinear Science and Numerical Simulation, vol. 32, pp. 211224, 2016.

[11] Z. Xing, Y. Xia, L. Yan, K. Lu, and Q. Gong, "Multisensor Distributed Weighted Kalman Filter Fusion With Network Delays, Stochastic Uncertainties, Autocorrelated, and CrossCorrelated Noises," IEEE Transactions on Systems, Man, and Cybernetics: Systems, no. 99, pp. 1-11, 2016.

[12] W. Liu, "Optimal Filtering for Discrete-Time Linear Systems with Time-Correlated Multiplicative Measurement Noises," IEEE Transactions on Automatic Control, vol. 61, no. 7, pp. 19721978, 2016.

[13] M. J. García-Ligero, A. Hermoso-Carazo, and J. Linares-Pérez, "Estimation from a multisensor environment for systems with multiple packet dropouts and correlated measurement noises," Applied Mathematical Modelling, vol. 45, pp. 802-812, 2017.

[14] H. Shu, S. Zhang, B. Shen, and Y. Liu, "Unknown input and state estimation for linear discrete-time systems with missing measurements and correlated noises," International Journal of General Systems, vol. 45, no. 5, pp. 648-661, 2016.

[15] S. Wang, H. Fang, and X. Tian, "Minimum variance estimation for linear uncertain systems with one-step correlated noises and incomplete measurements," Digital Signal Processing, vol. 49, pp. 126-136, 2016.

[16] R. Caballero-Águila, A. Hermoso-Carazo, and J. Linares-Pérez, "Networked fusion filtering from outputs with stochastic uncertainties and correlated random transmission delays," Sensors, vol. 16, no. 6, 2016.

[17] W.-Q. Liu, X.-M. Wang, and Z.-L. Deng, "Robust centralized and weighted measurement fusion Kalman estimators for uncertain multisensor systems with linearly correlated white noises," Information Fusion, vol. 35, pp. 11-25, 2017.

[18] N. Pletschen and K. J. Diepold, "Nonlinear state estimation for suspension control applications: a Takagi-Sugeno Kalman filtering approach," Control Engineering Practice, vol. 61, pp. 292-306, 2017.
[19] D. Ding, Y. Shen, Y. Song, and Y. Wang, "Recursive state estimation for discrete time-varying stochastic nonlinear systems with randomly occurring deception attacks," International Journal of General Systems, vol. 45, no. 5, pp. 548-560, 2016.

[20] Y. Sun, X. Wu, J. Cao, Z. Wei, and G. Sun, "Fractional extended Kalman filtering for non-linear fractional system with Lévy noises," IET Control Theory \& Applications, vol. 11, no. 3, pp. 349-358, 2017.

[21] L. Li, D. Yu, Y. Xia, and H. Yang, "Stochastic stability of a modified unscented Kalman filter with stochastic nonlinearities and multiple fading measurements," Journal of The Franklin Institute, vol. 354, no. 2, pp. 650-667, 2017.

[22] J. Devakumar and K. Srinivasan, "Improved state estimation for stochastic nonlinear chemical reactor using particle filter based on unscented transformation," Journal of Control Engineering and Applied Informatics, vol. 18, no. 4, pp. 650-667, 2016.

[23] H. Yan, F. Qian, F. Yang, and H. Shi, "Ho filtering for nonlinear networked systems with randomly occurring distributed delays, missing measurements and sensor saturation," Information Sciences, vol. 370-371, pp. 772-782, 2016.

[24] S. Zhang, J. Zhao, Y. Zhao, and G. Li, "Gain-Constrained Extended Kalman Filtering with Stochastic Nonlinearities and Randomly Occurring Measurement Delays," Circuits, Systems and Signal Processing, vol. 35, no. 11, pp. 3957-3980, 2016.

[25] Y. Liu, Z. Wang, X. He, and D. Hua, "Ho filtering for nonlinear systems with stochastic sensor saturations and Markov time delays: the asymptotic stability in probability," IET Control Theory \& Applications, vol. 10, no. 4, pp. 1706-1715, 2016.

[26] J. Mao, D. Ding, Y. Song, Y. Liu, and F. E. Alsaadi, "Event-based recursive filtering for time-delayed stochastic nonlinear systems with missing measurements," Signal Processing, vol. 134, pp. 158165, 2017. 


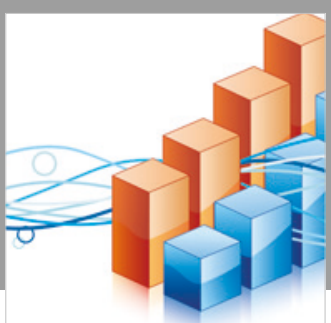

Advances in

Operations Research

\section{-n-m}
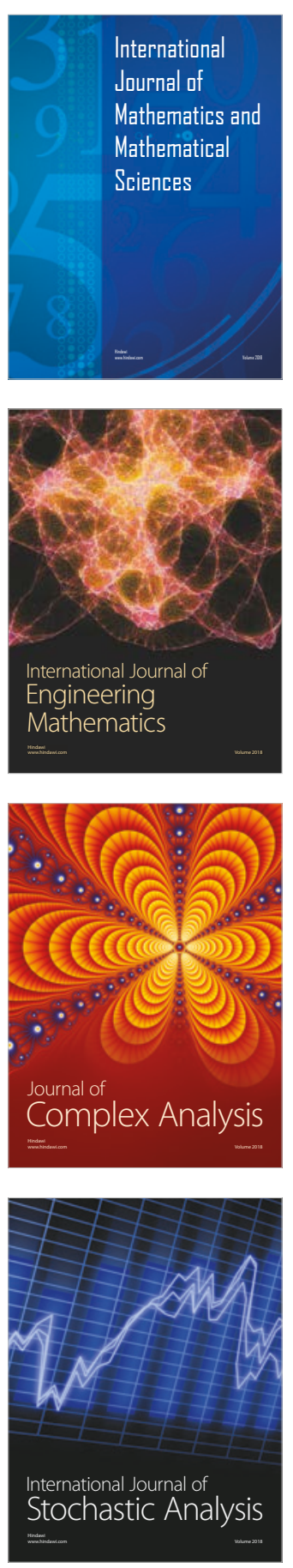
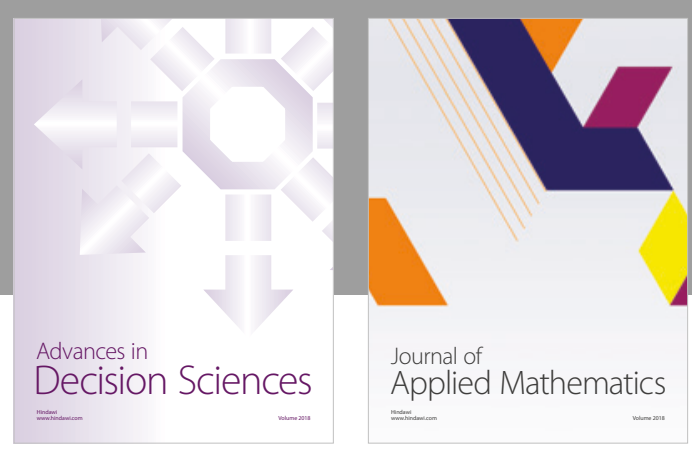

Journal of

Applied Mathematics
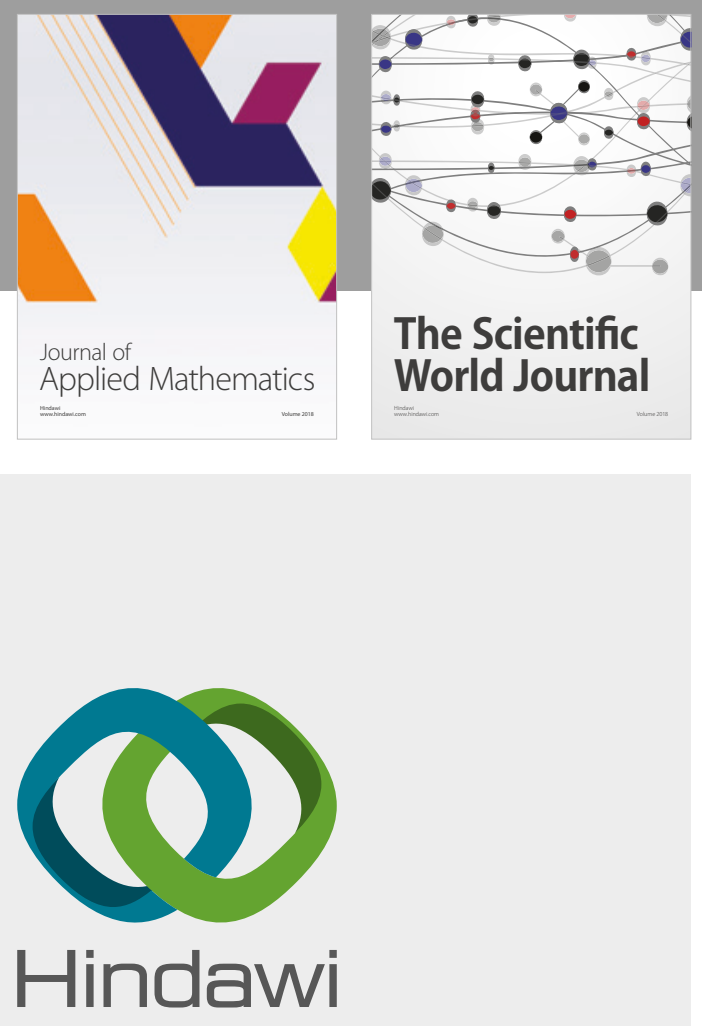

Submit your manuscripts at

www.hindawi.com

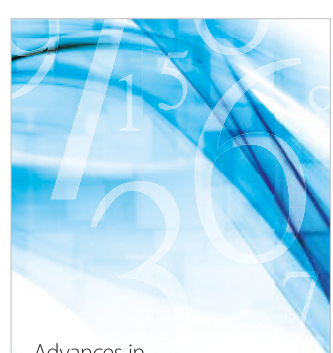

Advances in
Numerical Analysis
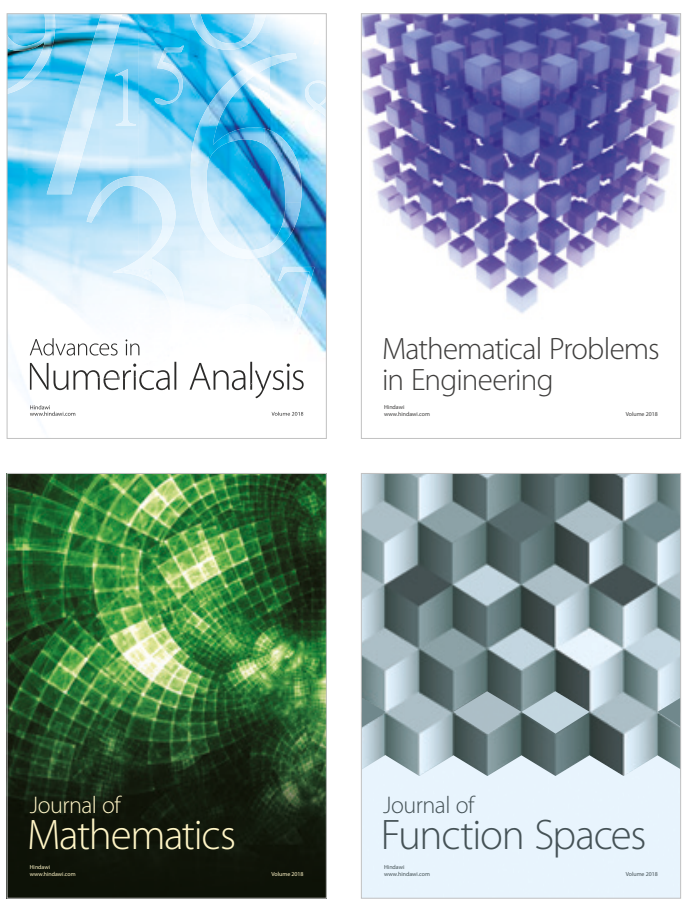

Mathematical Problems in Engineering

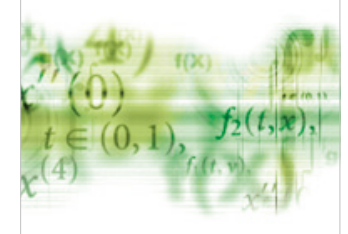

International Journal of

Differential Equations

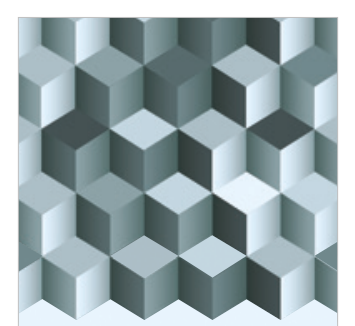

Journal of

Function Spaces

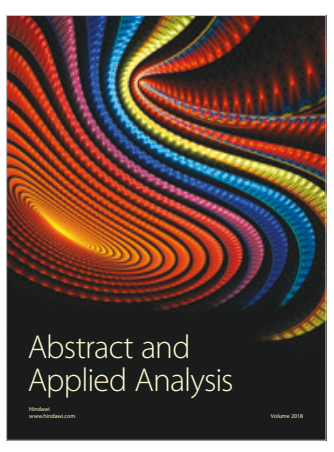

The Scientific

World Journal

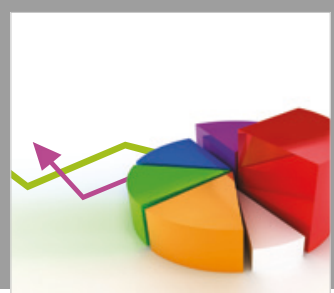

Journal of

Probability and Statistics
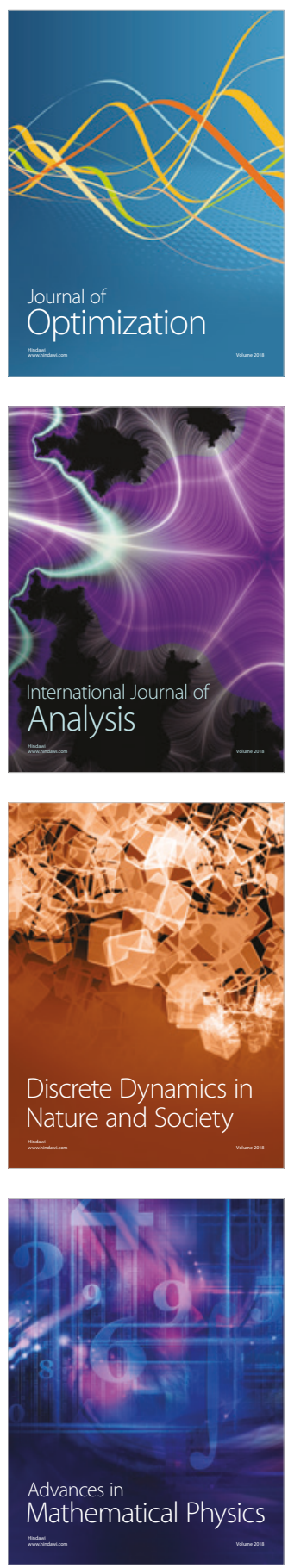